\title{
A review on arbitrarily regular conforming virtual element methods for second- and higher-order elliptic partial differential equations
}

\author{
Paola F. Antonietti* \\ MOX, Dipartimento di Matematica, \\ Politecnico di Milano, Italy \\ paola.antonietti@polimi.it \\ Gianmarco Manzini \\ IMATI, Consiglio Nazionale delle Ricerche, \\ Pavia, Italy \\ marco.manzini@imati.cnr.it \\ Simone Scacchi \\ Dipartimento di Matematica, \\ Università degli Studi di Milano, Italy \\ simone.scacchi@unimi.it \\ Marco Verani \\ MOX, Dipartimento di Matematica, \\ Politecnico di Milano, Italy \\ marco.verani@polimi.it
}

Received 22 March 2021

Revised 25 August 2021

Accepted 26 August 2021

Published 15 December 2021

Communicated by L. Beirão da Veiga, N. Bellomo, F. Brezzi and L.D. Marini

The virtual element method is well suited to the formulation of arbitrarily regular Galerkin approximations of elliptic partial differential equations of order $2 p_{1}$, for any integer $p_{1} \geq 1$. In fact, the virtual element paradigm provides a very effective design framework for conforming, finite dimensional subspaces of $H^{p_{2}}(\Omega), \Omega$ being the computational domain and $p_{2} \geq p_{1}$ another suitable integer number. In this review, we first present an abstract setting for such highly regular approximations and discuss the mathematical details of how we can build conforming approximation spaces with a global high-order regularity on $\Omega$. Then, we illustrate specific examples in the case of secondand fourth-order partial differential equations, that correspond to the cases $p_{1}=1$ and 2, respectively. Finally, we investigate numerically the effect on the approximation properties of the conforming highly-regular method that results from different choices

* Corresponding author. 
of the degree of continuity of the underlying virtual element spaces and how different stabilization strategies may impact on convergence.

Keywords: Virtual element methods; arbitrarily regular conforming approximation spaces; second- and higher-order elliptic PDEs.

AMS Subject Classification 2020: 65N22, 65N30, 65N12

\section{Introduction}

In the recent years, there has been an intensive research on numerical approximations of partial differential equations (PDEs) that can work on unstructured polygonal and polyhedral (polytopal) meshes. Such research activity has led to the design of several families of numerical discretizations for PDEs, as, for example, the polygonal/polyhedral finite element method, $\frac{67}{6}$ the mimetic finite difference method, 22 the virtual element method (VEM) $\frac{17}{\sqrt[17]{1}}$ the discontinuous Galerkin method on polygonal/polyhedral grids;,$\sqrt[7 / 36]{ }$ the hybrid discontinuous Galerkin method, $\underline{44}$ and the hybrid high-order method. $\stackrel{47}{ }$ Roughly speaking, all these methods are Galerkintype projection methods where the solution of a PDE is approximated in a finitedimensional space that is built upon an underlying mesh made of arbitrarily-shaped polytopal elements. In this sense, all such methods can be considered as a generalization of the finite element method that is formulated on classical simplicial and quadrilateral meshes.

In particular, the virtual element method, which is the focus of our paper, has been proven to be very successful in numerical modeling of scientific and engineering applications. The conforming VEM was first developed for second-order

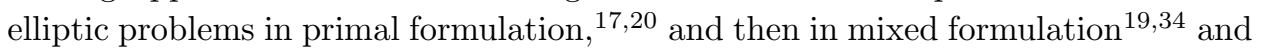
nonconforming formulation.15 A non-exhaustive list of applications includes the numerical approximation of underground flows and discrete fracture networks $29 \sqrt{30}$ propagation and scattering of time-harmonic waves, $57 \sqrt[56]{6}$ topology optimization problems $; \sqrt{60}$ contact mechanics and elasto-plastic deformation problems, $\frac{\sqrt[70]{40}}{\text { phase- }}$ field models of isotropic brittle fractures, $\frac{2}{2}$ the Schrödinger equation, $\frac{37}{\sqrt{37}}$ obstacle $\sqrt{68}$

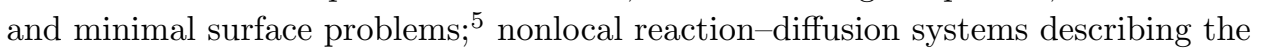
cardiac electric field, $\frac{\sqrt{3}}{3}$ cracks in materials, $\frac{31}{31}$ structural mechanics problems,,$\frac{13[14}{14}$ elastic wave propagation phenomena $\sqrt[86465]{6}$ The major reason of this success is that the VEM offers a great flexibility in designing approximation spaces featuring important properties other than just supporting polytopal meshes. Indeed, the VEM features great flexibility in dealing with internal constraints (e.g. locking phenomena) and in designing ad-hoc approximation spaces that preserve fundamental properties of the underlying physical and mathematical models (e.g. incompressibility constraint). It is worth mentioning the construction of virtual element spaces forming de Rham complexes for the Stokes equations, 24 the Navier-Stokes equations 25 and the Maxwell equations, $\frac{18}{18}$ where the numerical approximation of the velocity field or the magnetic flux field is pointwise divergence free. Another remarkable example is provided by the VEM for Helmholtz problems ${ }^{58}$ based on 
non-conforming approximation spaces of Trefftz functions, i.e. functions that belong to the kernel of the Helmholtz operator.

In this paper, we are interested in the construction of virtual element spaces with global arbitrarily high smoothness (regularity). We review the related literature in more detail in the next section as it is the central topic of the present study. High regularity of the numerical approximation is of primary importance when dealing with high-order differential problems, i.e. problems involving partial differential equations of order $2 p_{1}, p_{1} \geq 1$, and offers clear advantages even for $p_{1}=1$, i.e. in the context of second-order differential equations. Indeed, global smoothness can be useful to directly compute physical quantities (such as fluxes, strains, stresses) without resorting to post-processing as in classical $C^{0}$-continuous finite element method (FEM), to develop anisotropic error estimators based on the Hessian or to devise better eigenvalue approximation (studies in isogeometric analysis have shown that highly regular discrete spaces may give a better approximation of the high end of the spectrum). More precisely, the virtual element framework allows us to design finite-dimensional subspaces of $H^{p_{2}}(\Omega)$ for some suitable integer number $p_{2} \geq 1$. Here, the integer $p_{2}$ determines the global regularity of the virtual element functions defined on the computational domain $\Omega$. The value of $p_{2}$ obviously depends on the problem and the numerical approximation at hand and we will always assume that $p_{2} \geq p_{1}$.

In the "classical" conforming FEM, the finite-dimensional spaces are typically only $C^{0}$-continuous, $\frac{42}{2}$ and the definition of more regular approximation spaces is usually considered a difficult task from both the theoretical and computational viewpoints. The major difficulty in the formulation of a $C^{1}$-regular FEM relies in the explicit construction of a set of basis functions with such global regularity $12|28| 43$ More recent results on the construction of highly-regular finite element spaces can be found, e.g. in Refs. 71, 72, 51 and 50. The remarkable aspect that makes the VEM so appealing in this respect is that the formulation of such arbitrary regular approximations and their implementation are relatively straightforward. The crucial point here is that in the virtual element setting we do not need to know explicitly the shape functions spanning the virtual element space. All the virtual element functions are indeed virtual in the sense that they are implicitly defined as the solution of a local partial differential equation inside each mesh element. Consequently, such functions are not explicitly known, with the noteworthy exception of some subset of polynomials. Instead, they are uniquely defined by a set of values dubbed the degrees of freedom and these values are the only knowledge that we really need to formulate and implement the numerical scheme. This feature makes the construction of arbitrary regular approximations for any kind of partial differential equations much simpler and almost immediate.

Our first goal in this study is to provide a comprehensive overview of the stateof-the-art of highly regular conforming virtual element approximations of PDEs of order $2 p_{1}, p_{1} \geq 1$. Our second aim is to investigate the influence of different stabilization strategies on the performance of highly-regular virtual element discretizations 
in terms of the condition number of the resulting linear system of equations and accuracy of the approximation scheme. For the numerical validation, we focus on two model problems: the Poisson equation and the biharmonic equation in two spatial dimensions. In the next subsection, we provide an overview of the literature related to arbitrarily regular VEM discretizations.

\subsection{Background material on arbitrarily regular virtual element formulations}

The first work on a $C^{1}$-regular conforming VEM addressed the classical plate bending problem. ${ }^{35}$ In such a work, a $C^{1}$-regular virtual element method is proposed and analyzed for the numerical discretization of the Kirchhoff-Love model for thin plates. The approximation error is theoretically proved to decay in the energy norm, i.e. the $H^{2}$ norm, with the optimal rate $r-1, r \geq 2$, if the local virtual element spaces contain the space of polynomials of degree $r$. Optimal errors estimates in both $H^{1}$ and $L^{2}$ norms have been derived later using duality arguments $\frac{41}{\text { Suc- }}$ cessively, an arbitrarily regular virtual element approximation was developed for second-order elliptic problems in two dimensions by using similar concepts ${ }^{26}$ and then applied to the design of residual-based a-posteriori error estimators ${ }^{[27} \mathrm{A}$ loworder variant of this method was considered for the semi-discrete approximation of the two-dimensional nonlinear Cahn-Hilliard problem. ${ }^{4}$ Such VEM needs only three degrees of freedom per mesh vertex and turns out to be a new discretization also on triangular grids. Recently, highly regular virtual element spaces have also been considered for the numerical resolution of the von Kármán equation modeling the deformation of very thin plates ${ }^{[55}$ Here, the model under consideration is a fourthorder system of nonlinear partial differential equations where the unknowns describe the transverse displacement and the boundary stresses of the plate. The resulting conforming formulation is shown to be well-posed through a Banach fixed-point argument provided that the mesh size is small enough, and optimal errors bounds are proved when the error is measured in the $H^{2}$ norm. Interesting applications of highly-regular conforming VEM can be also found in the context of geostrophic equations ${ }^{61}$ and fourth-order subdiffusion equations. $\stackrel{54}{ }$ Highly-regular conforming VEMs have been recently proposed and analyzed for general polyharmonic boundary value problems $\frac{10}{}$ of the form $(-\Delta)^{p_{1}} u=f, p_{1} \geq 1$, in two dimensions. The virtual element space of this method contains polynomials of degree $r \geq 2 p_{1}-1$, features $H^{p_{1}}$ global regularity and guarantees optimal approximation bounds in suitable norms, i.e. with the above introduced notation it corresponds to the choice $p_{2}=p_{1}$. This approach is an extension of the known virtual element discretization of second- and fourth-order problems since the approximation spaces for $p_{1}=p_{2}=1$ and $p_{1}=p_{2}=2$ coincide with the conforming virtual element spaces for the Poisson equation $\frac{17}{17}$ and the biharmonic equation, $\frac{35}{35}$ respectively.

All the previously mentioned works focus onto two-dimensional mathematical models. The first highly regular VEM in the three-dimensional setting addresses 
the fourth-order linear elliptic equation 21 (see also Ref. 33). The lowest-order case requires a virtual element space locally including quadratic polynomials, i.e. $r=2$. The degrees of freedom are the values of the virtual element functions and their gradients at the mesh vertices. Recently, highly-regular conforming VEM in any dimension has been proposed in Ref. 53 .

A highly regular virtual element method has also been designed for solving the eigenvalue problem modeling the two-dimensional plate vibration problem of Kirchhoff plates. ${ }^{60}$ For the resulting spectral problem, the lowest-order $H^{2}(\Omega)$ conforming VEM provides the correct spectral approximation and optimal-order error estimates are derived for the approximation of the eigenvalues and the eigenfunctions. Along the same line, a fourth-order spectral problem derived from the transmission eigenvalue problem is considered in Ref. 62, Its variational formulation is written in $H^{2}(\Omega) \times H^{1}(\Omega)$ and the resulting virtual element approximation is $H^{2}(\Omega) \times H^{1}(\Omega)$-conforming. Employing the classical approximation theory for compact non-self-adjoint operators, it is shown that the resulting VEM provides a correct approximation of the spectrum, and the eigenvalues and eigenfunctions are approximated with the expected (optimal) rates. The fourth-order plate buckling eigenvalue problem has recently been addressed ${ }^{63}$ Here, a $C^{1}$-regular virtual element method of arbitrary order $r \geq 2$ is used to approximate the buckling coefficients and modes. This virtual element space is an extension of the approximation space introduced in Refs. 35] and 4. In view of the Babuška-Osborn abstract spectral approximation theory, $\sqrt{16}$ this VEM provides a correct approximation of the spectrum. Optimal-order error estimates for the buckling modes and the buckling coefficients are derived.

Finally, it is worth mentioning that in the context of fourth- or higher-order problems, alternative strategies based on non-conforming approaches are also viable and have been addressed in the recent literature. For example, for the biharmonic problem we find $C^{0}$ non-conforming $9^{73}$ and fully non-conforming ${ }^{974}$ virtual element approximations, and for higher-order PDEs in $\mathbb{R}^{n}$ we find non-conforming VEM 3952 A unified general framework including the lowest-order conforming $\mathrm{VEM}^{35}$ and non-conforming $\mathrm{VEM}^{9 / 73 / 74}$ has also been proposed and analyzed for the Kirchhoff plate contact problem with friction $\underline{69}$

\subsection{Outline of the paper}

The remaining part of the paper is organized as follows. In Sec. 2 , we introduce the continuous problem and its weak formulation. In Sec. 3, we introduce the virtual element discretization and recall the main abstract convergence result. In Sec. 4. we present the conforming virtual element approximation with higher-order regularity and recall the main theoretical results for polyharmonic problems. 10 Moreover, employing the ideas of Ref. 26, we extend to the case $r \geq p_{2}$ the construction of lower order spaces as considered in Ref. 10. Section 5 is devoted to present numerical experiments for second- and fourth-order elliptic PDEs. We assess the convergence 
properties of the VEM versus the mesh size and the degree of continuity of the underling virtual element space for different possible choices of the stabilization. We also investigate numerically how these choices impact on the condition number of the resulting linear system of equations. Finally, in Sec. 6, we draw our conclusions.

\section{The Continuous Problem}

In this section, we introduce the model problem under investigation together with its weak formulation. Let $\Omega \subset \mathbb{R}^{2}$ be an open, bounded, convex domain with polygonal boundary $\Gamma$. For any integer $p_{1} \geq 1$, we introduce the conforming virtual element method for the approximation of the following problem:

$$
\begin{aligned}
(-\Delta)^{p_{1}} u=f & \text { in } \Omega, \\
\partial_{n}^{j} u=0 & \text { for } j=0, \ldots, p_{1}-1 \text { on } \Gamma,
\end{aligned}
$$

where $\partial_{n}^{j} u$ is the normal derivative of order $j$ of the function $u$ with useful conventional notation that $\partial_{n}^{0} u=u$. Let

$$
V \equiv H_{0}^{p_{1}}(\Omega)=\left\{v \in H^{p_{1}}(\Omega): \partial_{n}^{j} v=0 \text { on } \Gamma, j=0, \ldots, p_{1}-1\right\} .
$$

Denoting the duality pairing between $V$ and its dual $V^{\prime}$ by $\langle\cdot, \cdot\rangle$, the variational formulation of the polyharmonic problem 2.1 reads as: Find $u \in V$ such that

$$
a_{p_{1}}(u, v)=\langle f, v\rangle \quad \forall v \in V
$$

where, for any nonnegative integer $\ell$, the bilinear form is given by:

$$
a_{p_{1}}(u, v)= \begin{cases}\int_{\Omega} \nabla^{\ell} u \cdot \nabla \Delta^{\ell} v d x & \text { for } p_{1}=2 \ell+1, \\ \int_{\Omega} \Delta^{\ell} u \Delta^{\ell} v d x & \text { for } p_{1}=2 \ell .\end{cases}
$$

Whenever $f \in L^{2}(\Omega)$ we have

$$
\langle f, v\rangle=(f, v)=\int_{\Omega} f v d x,
$$

where $(\cdot, \cdot)$ denotes the $L^{2}$-inner product. The existence and uniqueness of the solution to 2.2 follows from the Lax-Milgram Theorem because of the continuity and coercivity of the bilinear form $a_{p_{1}}^{\mathrm{P}}(\cdot, \cdot)$ with respect to $\|\cdot\|_{V}=|\cdot|_{p_{1}, \Omega}$, which is a norm on $H_{0}^{p_{1}}(\Omega)$. Moreover, whenever $\partial \Omega \in C^{k}$, for $k \geq 2 p_{1}$, from Corollary 2.21 in Ref. 48 we know that $u \in H^{k}(\Omega) \cap H_{0}^{p_{1}}(\Omega)$ if $f \in H^{k-2 p_{1}}(\Omega)$, and it holds that $\|u\|_{k} \leq C\|f\|_{k-2 p_{1}}$. In the case of a bounded, convex, polygonal domain, it is expected that similar results hold depending on the values of the internal angles (cf. Ref. 32 for the biharmonic case, i.e. $p_{1}=2$ ). In the following, we denote the coercivity and continuity constants of $a_{p_{1}}(\cdot, \cdot)$ by $\alpha$ and $M$, respectively. 
Let $\mathrm{P}$ be a polygonal element and denote by $a_{p_{1}}^{\mathrm{P}}(\cdot, \cdot)$ the restriction of $a_{p_{1}}(\cdot, \cdot)$ to $\mathrm{P}$. For an odd $p_{1}$, i.e. $p_{1}=2 \ell+1$, a repeated application of the integration by parts formula yields

$$
\begin{aligned}
a_{p_{1}}^{\mathrm{P}}(u, v)= & -\int_{\mathrm{P}} \Delta^{p_{1}} u v d x+\int_{\partial \mathrm{P}} \partial_{n}\left(\Delta^{\ell} u\right) \Delta^{\ell} v d s \\
& +\sum_{i=1}^{\ell}\left(\int_{\partial \mathrm{P}} \partial_{n}\left(\Delta^{p_{1}-i} u\right) \Delta^{i-1} v d s-\int_{\partial \mathrm{P}} \Delta^{p_{1}-i} u \partial_{n}\left(\Delta^{i-1} v\right) d s\right),
\end{aligned}
$$

while, for an even $p_{1}$, i.e. $p_{1}=2 \ell$, we have

$$
\begin{aligned}
a_{p_{1}}^{\mathrm{P}}(u, v) & =\int_{\mathrm{P}} \Delta^{p_{1}} u v d x \\
& =-\sum_{i=1}^{\ell}\left(\int_{\partial \mathrm{P}} \partial_{n}\left(\Delta^{p_{1}-i} u\right) \Delta^{i-1} v d s-\int_{\partial \mathrm{P}} \Delta^{p_{1}-i} u \partial_{n}\left(\Delta^{i-1} v\right) d s\right) .
\end{aligned}
$$

The above formulas will be crucial to prove the unisolvence of the degrees of freedom of the virtual element spaces and to show the computability of the elliptic projections (cf. Sec. 3).

\section{The Discrete Problem and Abstract Convergence Result}

In this section, we present the discrete counterpart of formulation 2.2 and recall the abstract convergence result. Let $\left\{\Omega_{h}\right\}_{h}$ be a sequence of decompositions of $\Omega$ where each mesh $\Omega_{h}$ is a collection of nonoverlapping polygonal elements $\mathrm{P}$ with boundary $\partial \mathrm{P}$, and let $\mathcal{E}_{h}$ be the set of edges $e$ of $\Omega_{h}$. Each mesh is labeled by $h$, the diameter of the mesh, defined as usual by $h=\max _{\mathrm{P} \in \Omega_{h}} h_{\mathrm{P}}$, where $h_{\mathrm{P}}=$ $\sup _{x, y \in \mathrm{P}}|\mathbf{x}-\mathbf{y}|$. We denote the set of vertices in $\Omega_{h}$ by $\mathcal{V}_{h}$. The symbol $h_{\mathrm{v}}$ denotes the average of the diameters of the polygons sharing the vertex v. For functions in $\Pi_{\mathrm{P} \in \Omega_{h}} H^{p_{1}}(\mathrm{P})$, we define the seminorm $\|v\|_{h}^{2}=\sum_{\mathrm{P} \in \Omega_{h}} a_{p_{1}}^{\mathrm{P}}(v, v)$.

The formulation of the virtual element method for the approximation of the solution to the elliptic problem 2.2 with arbitrarily smooth functions only requires three mathematical objects:

(1) for $p_{2} \geq p_{1} \geq 1$ the finite-dimensional conforming virtual element space $V_{h, r}^{p_{2}, p_{1}} \subset H_{0}^{p_{1}}(\Omega) \cap H^{p_{2}}(\Omega) \subset V$

(2) the bilinear form $a_{p_{1}, h}(\cdot, \cdot)$;

(3) the linear functional $\left\langle f_{h}, \cdot\right\rangle$.

Note that the space $V_{h, r}^{p_{2}, p_{1}}$ is made of globally $H^{p_{2}}(\Omega)$ functions and, endowed with suitable degrees of freedom, will be employed to solve elliptic problems of order $p_{1} \leq p_{2}$. 
Using such objects, we formulate the VEM as: Find $u_{h} \in V_{h, r}^{p_{2}, p_{1}}$ such that

$$
a_{p_{1}, h}\left(u_{h}, v_{h}\right)=\left\langle f_{h}, v_{h}\right\rangle \quad \forall v_{h} \in V_{h, r}^{p_{2}, p_{1}} .
$$

The well-posedness of (3.1), which implies existence and uniqueness of the solution $u_{h}$, is a consequence of the Lax-Milgram lemma. An abstract convergence result is available, which depends only on the following assumptions:

(H1) for each $h$ and an assigned integer number $r \geq p_{2}$ we are given:

(1) the global virtual element space $V_{h, r}^{p_{2}, p_{1}}$ with the following properties:

- $V_{h, r}^{p_{2}, p_{1}}$ is a finite-dimensional subspace of $H_{0}^{p_{1}}(\Omega) \cap H^{p_{2}}(\Omega) \subset V$;

- its restriction $V_{h, r}^{p_{2}, p_{1}}(\mathrm{P})$ to any element $\mathrm{P}$ of a given mesh $\Omega_{h}$, called the local (elemental) virtual element space, is a finite-dimensional subspace of $H^{p_{2}}(\mathrm{P})$;

- $\mathbb{P}_{r}(\mathrm{P}) \subset V_{h, r}^{p_{2}, p_{1}}(\mathrm{P})$ where $\mathbb{P}_{r}(\mathrm{P})$ is the space of polynomials of degree up to $r$ defined on $\mathrm{P}$;

(2) the symmetric bilinear form $a_{p_{1}, h}: V_{h, r}^{p_{2}, p_{1}} \times V_{h, r}^{p_{2}, p_{1}} \rightarrow \mathbb{R}$ admitting the decomposition

$$
a_{p_{1}, h}\left(u_{h}, v_{h}\right)=\sum_{\mathrm{P} \in \Omega_{h}} a_{p_{1}, h}^{\mathrm{P}}\left(u_{h}, v_{h}\right) \quad \forall u_{h}, \quad v_{h} \in V_{h, r}^{p_{2}, p_{1}},
$$

where each local summation term $a_{p_{1}, h}^{\mathrm{P}}(\cdot, \cdot)$ is also a symmetric bilinear form;

(3) an element $f_{h}$ of the dual space $\left(V_{h, r}^{p_{2}, p_{1}}\right)^{*}$ of $V_{h, r}^{p_{2}, p_{1}}$, which allows us to define the continuous linear functional $\left\langle f_{h}, \cdot\right\rangle$.

(H2) for each $h$ and each mesh element $\mathrm{P} \in \Omega_{h}$, the local symmetric bilinear form $a_{p_{1}, h}^{\mathrm{P}}(\cdot, \cdot)$ possesses the two following properties:

(i) $r$-Consistency: for every polynomial $q \in \mathbb{P}_{r}(\mathrm{P})$ and virtual element function $v_{h} \in V_{h, r}^{p_{2}, p_{1}}(\mathrm{P})$ it holds

$$
a_{p_{1}, h}^{\mathrm{P}}\left(v_{h}, q\right)=a_{p_{1}}^{\mathrm{P}}\left(v_{h}, q\right)
$$

(ii) Stability: there exist two positive constants $\alpha_{*}, \alpha^{*}$ independent of $h$ and $\mathrm{P}$ such that for every $v_{h} \in V_{h, r}^{p_{2}, p_{1}}(\mathrm{P})$ it holds

$$
\alpha_{*} a_{p_{1}}^{\mathrm{P}}\left(v_{h}, v_{h}\right) \leq a_{p_{1}, h}^{\mathrm{P}}\left(v_{h}, v_{h}\right) \leq \alpha^{*} a_{p_{1}}^{\mathrm{P}}\left(v_{h}, v_{h}\right) .
$$

It is easy to check that $a_{p_{1}, h}(\cdot, \cdot)$ is coercive and continuous. Let $\mathbb{P}_{r}\left(\Omega_{h}\right)$ denote the space of piecewise (possibly discontinuous) polynomials defined over the mesh $\Omega_{h}$. The following abstract convergence result holds.

Theorem 3.1. Let $u$ be the solution of the variational problem 2.2. Then, for every virtual element approximation $u^{I}$ in $V_{h, r}^{p_{2}, p_{1}}$ and any piecewise polynomial approximation $u_{\pi} \in \mathbb{P}_{r}\left(\Omega_{h}\right)$ of $u$ we have

$$
\left\|u-u_{h}\right\|_{\vee} \leq C\left(\left\|u-u^{I}\right\|_{v}+\left\|u-u_{\pi}\right\|_{h}+\left\|f_{h}-f\right\|_{\left(V_{h, r}^{p_{2}, p_{1}}\right)^{*}}\right),
$$


where $C$ is a constant independent of $h$ that may depend on $\alpha, M, \alpha_{*}, \alpha^{*}$, and $r$, and

$$
\left\|f-f_{h}\right\|_{\left(V_{h, r}^{p_{2}, p_{1}}\right)^{*}}=\sup _{v_{h} \in V_{h, r}^{p_{2}, p_{1}} \backslash\{0\}} \frac{\left\langle f-f_{h}, v_{h}\right\rangle}{\left\|v_{h}\right\|_{v}}
$$

is the approximation error of the right-hand side given in the norm of the dual space $\left(V_{h, r}^{p_{2}, p_{1}}\right)^{*}$.

Proof. We report here the proof for completeness. ${ }^{10}$ First, an application of the triangular inequality implies that

$$
\left\|u-u_{h}\right\|_{\vee} \leq\left\|u-u^{I}\right\|_{\vee}+\left\|u^{I}-u_{h}\right\|_{v} .
$$

Let $\delta_{h}=u_{h}-u^{I}$ and recall that $\alpha$ is the coercivity constant of $a_{p_{1}}(\cdot, \cdot)$. Starting from the definition of $\|\cdot\|_{v}$, we find that

$$
\begin{aligned}
& \alpha_{*} \alpha\left\|\delta_{h}\right\|_{v}^{2} \leq \alpha_{*} a_{p_{1}}\left(\delta_{h}, \delta_{h}\right) \\
& \leq a_{p_{1}, h}\left(\delta_{h}, \delta_{h}\right) \\
& \leq a_{p_{1}, h}\left(\delta_{h}, u_{h}\right)-a_{p_{1}, h}\left(\delta_{h}, u^{I}\right) \\
& \leq\left\langle f_{h}, \delta_{h}\right\rangle-\sum_{\mathrm{P} \in \Omega_{h}} a_{p_{1}, h}^{\mathrm{P}}\left(\delta_{h}, u^{I}\right) \\
& \leq\left\langle f_{h}, \delta_{h}\right\rangle-\sum_{\mathrm{P} \in \Omega_{h}}\left(a_{p_{1}, h}^{\mathrm{P}}\left(\delta_{h}, u^{I}-u_{\pi}\right)+a_{p_{1}, h}^{\mathrm{P}}\left(\delta_{h}, u_{\pi}\right)\right) \\
& \leq\left\langle f_{h}, \delta_{h}\right\rangle-\sum_{\mathrm{P} \in \Omega_{h}}\left(a_{p_{1}, h}^{\mathrm{P}}\left(\delta_{h}, u^{I}-u_{\pi}\right)+a_{p_{1}}^{\mathrm{P}}\left(\delta_{h}, u_{\pi}\right)\right) \\
& \leq\left\langle f_{h}, \delta_{h}\right\rangle-\sum_{\mathrm{P} \in \Omega_{h}} \\
& \times\left(a_{p_{1}, h}^{\mathrm{P}}\left(\delta_{h}, u^{I}-u_{\pi}\right)+a_{p_{1}}^{\mathrm{P}}\left(\delta_{h}, u_{\pi}-u\right)+a_{p_{1}}^{\mathrm{P}}\left(\delta_{h}, u\right)\right) \\
& =\left\langle f_{h}-f, \delta_{h}\right\rangle-\sum_{\mathrm{P} \in \Omega_{h}}\left(a_{p_{1}, h}^{\mathrm{P}}\left(\delta_{h}, u^{I}-u_{\pi}\right)+a_{p_{1}}^{\mathrm{P}}\left(\delta_{h}, u_{\pi}-u\right)\right) . \\
& \text { [add } \pm u_{\pi} \text { ] } \\
& \text { [add } \pm u \text { ] }
\end{aligned}
$$

Then, we use (3.3), add and subtract $u$, use the continuity of $a_{p_{1}}^{\mathrm{P}}$, sum over all the elements $\mathrm{P}$, divide by $\left\|\delta_{h}\right\|_{\mathrm{v}}$, take the supremum of the right-hand side error term on $V_{h, r}^{p_{2}, p_{1}} \backslash\{0\}$, and obtain

$$
\alpha \alpha_{*}\left\|\delta_{h}\right\|_{\vee} \leq \sup _{v_{h} \in V_{h, r}^{p_{2}, p_{1}} \backslash\{0\}} \frac{\left|\left\langle f_{h}-f, v_{h}\right\rangle\right|}{\left\|v_{h}\right\|_{\vee}}+M\left(\alpha^{*}\left\|u^{I}-u\right\|_{v}+\left(1+\alpha^{*}\right)\left\|u-u_{\pi}\right\|_{h}\right),
$$

where $M$ is the continuity constant of $a_{p_{1}}(\cdot, \cdot)$. The assertion of the theorem follows by substituting 3.7 in 3.6 and suitably defining the constant $C$.

From the proof of Theorem 3.1 it follows that the constant $C$ appearing in (3.4) scales as $\frac{M}{\alpha} \frac{\alpha^{*}}{\alpha_{*}}$, where $\alpha$ and $M$ are the coercivity and continuity constants of $a_{p_{1}}(\cdot, \cdot)$ (in this case $\alpha=M=1$ ) whereas $\alpha^{*}$ and $\alpha_{*}$ are the constants of (H2), see 3.3. We point out that $\alpha^{*}$ and $\alpha_{*}$ might depend on the polynomial approximation degree $r$, see e.g. Ref. 11 for the case $p_{1}=1$. 


\section{The Virtual Element Spaces of Higher-Order Continuity}

\subsection{Preliminaries}

The "degrees of freedom tuples" are a very effective way to characterize the set of degrees of freedom (dofs) that uniquely identify the virtual element functions as members of a finite dimensional subspace of a $C^{k}$-regular virtual element space. Our degrees of freedom tuple, abbreviated as "dofs-tuple", is a generalization of the similar concept that was originally introduced for the degrees of freedom of a nonconforming virtual element space! ${ }^{46}$ Our dofs-tuple is an array $M_{k} \in \mathbb{Z}^{2(k+1)+1}$ defined by

$$
M_{k}=\left(\left(d_{0}^{v}, \ldots d_{k}^{v}\right),\left(d_{0}^{e}, \ldots, d_{k}^{e}\right), d_{0}^{i}\right)
$$

The integer variables $\left(d_{j}^{v}\right)$ and $\left(d_{j}^{e}\right)$, for $j=0, \ldots, k$, respectively encode the information associated with the mesh vertices and mesh edges; the last integer variable $d_{0}^{i}$ encodes the information associated with the interior of the mesh elements $\mathrm{P}$. The subscript $j=0$ in $d_{0}^{\vee}, d_{0}^{e}$, and $d_{0}^{i}$ indicates that these variables refer to the virtual element function. The subscript values $j=1, \ldots, k$ in $d_{j}^{\mathrm{v}}$ and $d_{j}^{e}$ denote the reference to the partial derivatives $D^{\nu}=\partial^{|\nu|} / \partial x^{\nu_{1}} \partial y^{\nu_{2}}$ of order $|\nu|=\nu_{1}+\nu_{2}=j$ of the virtual element function $\left(\nu=\left(\nu_{1}, \nu_{2}\right)\right.$ being a multi-index). The vertex variables $d_{j}^{v}$ can only take the values -1 or 0 , while the edge variables $d_{j}^{e}$ and the elemental variable $d_{0}^{i}$ either take the value -1 or a nonnegative integer value. If the entry is equal to -1 , the corresponding term is not used as a degree of freedom. If $d_{j}^{v}=0$, the $j$ th order partial derivatives evaluated at the mesh vertices are in the set of degrees of freedom (with the usual convention that $D^{\nu} v_{h}(\mathrm{v})=v_{h}(\mathrm{v})$ for $\nu=(0,0)$, i.e. $j=0)$. If $d_{j}^{\vee}=-1$, the corresponding $j$ th-order partial derivatives at the vertices are not degrees of freedom; this is typical of nonconforming methods (cf. Ref. 46). A nonnegative value of $d_{0}^{e}$ and $d_{0}^{i}$ defines the maximum order of the polynomial moments used in the definition of the degrees of freedom associated with the elemental edges $e \in \partial \mathrm{P}$ and the interior of the element $\mathrm{P}$.

By using the dofs-tuple $M_{k}$, we define the following set of values of a function $v \in H^{k+1}(\mathrm{P})$ :

(D1) $h_{\mathrm{v}}^{|\nu|} D^{\nu} v_{h}(\mathrm{v})$ at all vertices $\vee$ of the polygonal boundary $\partial \mathrm{P}$, for every multiindex $\nu=\left(\nu_{1}, \nu_{2}\right)$ such that $|\nu|=j$ if $d_{j}^{\mathrm{v}}=0, j=0, \ldots, k$;

(D2) $h_{e}^{-1+j} \int_{e} q \partial_{n}^{j} v_{h} d s$ for any $q \in \mathbb{P}_{d_{j}^{e}}(e), j=0, \ldots, k$ and any edge $e$ of $\partial \mathrm{P}$;

(D3) $h_{\mathrm{P}}^{-2} \int_{\mathrm{P}} q_{h} v_{h} d \mathbf{x}$ for any $q \in \mathbb{P}_{d_{0}^{i}}(\mathrm{P})$.

\subsection{Local and global spaces}

For $p_{2} \geq p_{1} \geq 1$ we first consider the case $r \geq 2 p_{2}-1$, while the lower order case $p_{2} \leq r \leq 2 p_{2}-1$ will be addressed in Sec. 4.3 . The local virtual element space on 
element $\mathrm{P}$ is defined by

$$
\begin{array}{r}
V_{h, r}^{p_{2}, p_{1}}(\mathrm{P})=\left\{v_{h} \in H^{p_{2}}(\mathrm{P}): \Delta^{p_{2}} v_{h} \in \mathbb{P}_{r-2 p_{1}}(\mathrm{P}), \partial_{n}^{i} v_{h} \in \mathbb{P}_{r-i}(e),\right. \\
\left.i=0, \ldots, p_{2}-1 \forall e \in \partial \mathrm{P}\right\},
\end{array}
$$

with the conventional notation that $\mathbb{P}_{r}(\mathrm{P})=\{0\}$ if $r<0$. The virtual element space $V_{h, r}^{p_{2}, p_{1}}(\mathrm{P})$ contains the space of polynomials $\mathbb{P}_{r}(\mathrm{P})$, for $r \geq 2 p_{2}-1$.

We take $k=p_{2}-1$ in 4.1 and endow the local space $V_{h, r}^{p_{2}, p_{1}}(\mathrm{P})$ with the dofs-tuple $M_{p_{2}-1}=M_{p_{2}-1}\left(p_{1}\right)$, which depends on the parameter $p_{1}$ by setting

$$
\begin{aligned}
& d_{j}^{\vee}=0 \quad j=0, \ldots, p_{2}-1, \\
& d_{j}^{e}=r-2 p_{2}+j \quad j=0, \ldots, p_{2}-1, \\
& d_{0}^{i}=r-2 p_{1} .
\end{aligned}
$$

Employing (2.5)-2.6) it is possible to prove that the degrees of freedom (D1)(D3) defined through the dofs-tuple $M_{p_{2}-1}\left(p_{1}\right)$ are unisolvent in $V_{h, r}^{p_{2}, p_{1}}(\mathrm{P})$, see Ref. 10. The particular choice of $d_{0}^{i}$ is essential for the computability of the elliptic projection with respect to $a_{p_{1}}^{\mathrm{P}}(\cdot, \cdot)$, which is a scalar product in $H_{0}^{p_{1}}(\Omega) \cap H^{p_{2}}(\Omega)$ cf. Remark 4.3 .

Building upon the local spaces $V_{h, r}^{p_{2}, p_{1}}(\mathrm{P})$ for all $\mathrm{P} \in \Omega_{h}$, the global conforming virtual element space $V_{h, r}^{p_{2}, p_{1}}$ is defined on $\Omega$ as

$$
V_{h, r}^{p_{2}, p_{1}}=\left\{v_{h} \in H_{0}^{p_{1}}(\Omega) \cap H^{p_{2}}(\Omega): v_{h \mid \mathrm{P}} \in V_{h, r}^{p_{2}, p_{1}}(\mathrm{P}) \forall \mathrm{P} \in \Omega_{h}\right\} .
$$

The set of global degrees of freedom inherited by the local degrees of freedom defined by $M_{p_{2}-1}\left(p_{1}\right)$ are

- $h_{\mathrm{v}}^{|\nu|} D^{\nu} v_{h}(\mathrm{v}),|\nu| \leq p_{2}-1$ for every interior vertex $\vee$ of $\Omega_{h}$;

- $h_{e}^{-1+j} \int_{e} q \partial_{n}^{j} v_{h} d s$ for any $q \in \mathbb{P}_{r-2 p_{2}+j}(e) j=0, \ldots, p_{2}-1$ and every interior edge $e \in \mathcal{E}_{h}$;

- $h_{\mathrm{P}}^{-2} \int_{\mathrm{P}} q v_{h} d \mathbf{x}$ for any $q \in \mathbb{P}_{r-2 p_{1}}(\mathrm{P})$ and every $\mathrm{P} \in \Omega_{h}$.

We remark that the associated global space is made of $H^{p_{2}}(\Omega)$ functions. Indeed, the restriction of a virtual element function $v_{h}$ to each element $\mathrm{P}$ belongs to $H^{p_{2}}(\mathrm{P})$ and glues with $C^{p_{2}-1}$-regularity across the internal mesh faces.

Remark 4.1. (Examples) We report some relevant examples from the virtual element literature that are included in the above abstract framework:

- for $p_{1}=p_{2}=1$, we obtain the $C^{0}$-conforming virtual element space for the Poisson equation, $\frac{17}{17}$

- for $p_{2}=p_{1}=2$ we obtain the conforming virtual element space for the biharmonic equation, $4 \sqrt[35]{35}$

- for $p_{1}=1$ and $p_{2}=2$ we obtain the $C^{1}$-conforming virtual element space for the Poisson equation, 26 
- for $p_{1}=1$ and $p_{2}=3$ we obtain the $C^{2}$-conforming virtual element space for the Poisson equation. 26

\subsection{Lower-order virtual spaces}

Following Ref. 26, lower-order elemental spaces can be defined that contains the subspace of polynomials of degree up to $r$ with $p_{2} \leq r \leq 2 p_{2}-2$ :

$$
\begin{aligned}
V_{h, r}^{p_{2}, p_{1}}(\mathrm{P})= & \left\{v_{h} \in H^{p_{2}}(\mathrm{P}): \Delta^{p_{2}} v_{h} \in \mathbb{P}_{r-2 p_{1}}(\mathrm{P}), \partial_{n}^{i} v_{h} \in \mathbb{P}_{\alpha_{i}}(e),\right. \\
& \left.i=0, \ldots, p_{2}-1 \forall e \in \partial \mathrm{P}\right\},
\end{aligned}
$$

where $\alpha_{j}=\max \left\{2 p_{2}-1-2 j, r-j\right\}$. Let $\beta_{j}=\alpha_{j}-\min \left\{2 p_{2}-1-2 j, r-j\right\}-1$.

For $r=2 p_{2}-1-k$ with $k=1, \ldots, p_{2}-1$, the virtual element functions in the elemental space 4.4 are uniquely identified by the degrees of freedom of the dofs-tuple $M_{p_{2}-1}\left(p_{1}\right)$ by setting

$$
\begin{aligned}
d_{j}^{\vee} & =0 \quad j=0, \ldots, p_{2}-1, \\
d_{j}^{e} & =-1 \quad j=0, \ldots, k, \\
d_{j}^{e} & =\beta_{j} \quad j=k+1, \ldots, p_{2}-1, \\
d_{0}^{i} & =r-2 p_{1} .
\end{aligned}
$$

Equivalently,

(D1) $h_{\vee}^{|\nu|} D^{\nu} v_{h}(\mathrm{v}),|\nu| \leq p_{2}-1$ for any vertex $\vee$ of $\partial \mathrm{P}$;

(D2) $h_{e}^{-1+j} \int_{e} q \partial_{n}^{j} v_{h} d s$ for any $q \in \mathbb{P}_{\beta_{j}}(e)$ and edge $e$ of $\partial \mathrm{P}, j=k+1, \ldots, p_{2}-1$; (D3) $h_{\mathrm{P}}^{-2} \int_{\mathrm{P}} q v_{h} d \mathbf{x}$ for any $q \in \mathbb{P}_{r-2 p_{1}}(\mathrm{P})$ and every $\mathrm{P} \in \Omega_{h}$.

Note that $\alpha_{j}=2 p_{2}-1-2 j$ for $j=0, \ldots, k$ and the degrees of freedom (D1) are sufficient to uniquely identify $\left.\partial_{n}^{j} v_{h}\right|_{e} \in \mathbb{P}_{2 p_{2}-1-2 j}(e)$ for $j=0, \ldots, p_{2}-1$; this motivates the choice $d_{j}^{e}=-1, j=0, \ldots, k$. The degrees of freedom (D2) are added to ensure $\left.\partial_{n}^{j} v_{h}\right|_{e} \in \mathbb{P}_{r-j}(e)$ whenever $\alpha_{j}=r-j>2 p_{2}-1-2 j$.

The above set of degrees of freedom is unisolvent in $V_{h, r}^{p_{2}, p_{1}}(\mathrm{P})$ and allows for the computability of the elliptic projection $\Pi_{r}^{p_{1}, \mathrm{P}}$ with respect to $a_{p_{1}}^{\mathrm{P}}(\cdot, \cdot)$. The global virtual element space $V_{h, r}^{p_{2}, p_{1}}$ is built as in the previous section and is made of $H^{p_{2}}$ functions.

Remark 4.2. The virtual space $V_{h, r}^{p_{2}, p_{1}}(\mathrm{P})$ in 4.4 for $r=2 p_{2}-2$ has been first introduced in the work of Ref. 10, while the virtual element spaces for $p_{2} \leq r<$ $2 p_{2}-2$ are new. The proofs of the dofs-unisolvence and of the computability of $\Pi_{r}^{p_{1}, \mathrm{P}}$ follow using the arguments of Ref. [10. In the lowest order case $\left(r=p_{2}\right)$ the local virtual element space $V_{h, r}^{p_{2}, p_{1}}(\mathrm{P})$ does not employ the dofs defined in (D2), so the corresponding dofs-tuple is equal to

$$
M_{p_{2}-1}\left(p_{1}\right)=\left(0, \ldots, 0,-1, \ldots,-1, d_{0}^{i}\right) .
$$


In particular, for $p_{1}=p_{2}=2$ and $r=2$ we obtain the space introduced in Ref. 4 for the conforming approximation of the Cahn-Hilliard equation. For $p_{1}=1$ and $r \geq p_{2} \geq 2$ we obtain the spaces introduced in Ref. 26 for the virtual element approximation of the Laplace problem with arbitrary regularity. The space $V_{h, p_{2}}^{p_{2}, p_{1}}(\mathrm{P})$ with $p_{1}=1,2$ will be employed in Sec. 5 to perform numerical tests. Finally, we note that $r \geq 2 p_{2}-1$ implies $\alpha_{j}=r-j$ and 4.4 reduces to 4.2.

\subsection{Projection operators and discrete bilinear forms}

The choice of $d_{0}^{i}$ in the dofs-tuple $M_{p_{2}-1}\left(p_{1}\right)$ is crucial for the computability of the elliptic projection $\Pi_{r}^{p_{1}, \mathrm{P}}: V_{h, r}^{p_{2}, p_{1}}(\mathrm{P}) \rightarrow \mathbb{P}_{r}(\mathrm{P})$, with respect to $a_{p_{1}}^{\mathrm{P}}(\cdot, \cdot)$. This fact will become clear in the discussion (see Remark 4.3). To define the elliptic projection we need the vertex average projector $\widehat{\Pi}^{\mathrm{P}}: V_{h, r}^{p_{2}, p_{1}}(\mathrm{P}) \rightarrow \mathbb{P}_{0}(\mathrm{P})$, which projects any (smooth enough) function defined on $\mathrm{P}$ onto the space of constant polynomials. Let $\psi$ be a continuous function defined on $\mathrm{P}$. The vertex average projection of $\psi$ onto the constant polynomial space is given by

$$
\widehat{\Pi}^{\mathrm{P}} \psi=\frac{1}{N^{\mathcal{P}}} \sum_{\mathrm{v} \in \partial \mathrm{P}} \psi(\mathrm{v}) .
$$

The elliptic projection $\Pi_{r}^{p_{1}, \mathrm{P}}: V_{h, r}^{p_{2}, p_{1}}(\mathrm{P}) \rightarrow \mathbb{P}_{r}(\mathrm{P})$ is the solution of the finitedimensional variational problem

$$
\begin{array}{rlrl}
a_{p_{1}}^{\mathrm{P}}\left(\Pi_{r}^{p_{1}, \mathrm{P}} v_{h}, q\right) & =a_{p_{1}}^{\mathrm{P}}\left(v_{h}, q\right) & & \forall q \in \mathbb{P}_{r}(\mathrm{P}), \\
\widehat{\Pi}^{\mathrm{P}} D^{\nu} \Pi_{r}^{p_{1}, \mathrm{P}} v_{h}=\widehat{\Pi}^{\mathrm{P}} D^{\nu} v_{h} & |\nu| \leq p_{1}-1 .
\end{array}
$$

Employing 2.5-2.6), in Ref. 10 it is shown that such operator has two important properties:

(i) it is polynomial-preserving in the sense that $\Pi_{r}^{p_{1}, \mathrm{P}} q=q$ for every $q \in \mathbb{P}_{r}(\mathrm{P})$;

(ii) the polynomial projection $\Pi_{r}^{p_{1}, \mathrm{P}} v_{h}$ is computable using only the degrees of freedom of $v_{h} \in V_{h, r}^{p_{2}, p_{1}}(\mathrm{P})$ that are specified by the dofs-tuple $M_{p_{2}-1}\left(p_{1}\right)$.

It is worth mentioning that the conditions in 4.7 can be replaced, for instance, by

$$
\int_{\partial \mathrm{P}}\left(\Pi_{r}^{p_{1}, \mathrm{P}} v_{h}-v_{h}\right) q d s=0 \quad \forall q \in \mathbb{P}_{p_{1}-1}(\mathrm{P}) .
$$

This latter equation has the technical advantage of allowing for the application to $\left.\left(I-\Pi_{r}^{p_{1}, \mathrm{P}}\right)\right|_{\mathrm{P}}$ of Poincaré type inequalities, which can be useful in proving approximation properties of $\Pi_{r}^{p_{1}, \mathrm{P}}$ in lower order norms.

Remark 4.3. (On the role of $d_{0}^{i}$ in the computability of $\Pi_{r}^{p_{1}, \mathrm{P}}$ ) We report a simple, but instructive example to clarify that the computability of $\Pi_{r}^{p_{1}}, \mathrm{P}$ is related to the interplay between the parameter $p_{1}$ (dictating the scalar product employed in the definition of the elliptic projection) and the degrees of freedom specified by dofs-tuple $M_{p_{2}-1}\left(p_{1}\right)$. 
For $p_{1}=1$ and $p_{2}=2, v_{h} \in V_{h, r}^{p_{2}, p_{1}}(\mathrm{P})$ and $q \in \mathbb{P}_{r}$, we have that

$$
a_{p_{1}}^{\mathrm{P}}\left(v_{h}, q\right)=\int_{\mathrm{P}} \nabla v_{h} \cdot \nabla q d \mathbf{x}=-\int_{\mathrm{P}} v_{h} \Delta q d \mathbf{x}+\int_{\partial \mathrm{P}} v_{h} \partial_{n} q d s .
$$

As $\Delta q \in \mathbb{P}_{r-2}$, the first term in the last equality on the right is computable in view of the choice of the degrees of freedom (D3) with $d_{0}^{i}=r-2 p_{1}=r-2$. The computability of the second term follows from the fact that the trace of $v_{h}$ on each edge of $\mathrm{P}$ is a polynomial that can be computed explicitly by interpolating the values in (D1) and (D2).

Now, we introduce the symmetric bilinear form $a_{h}: V_{h, r}^{p_{2}, p_{1}} \times V_{h, r}^{p_{2}, p_{1}} \rightarrow \mathbb{R}$, which is written as the sum of local terms

$$
a_{p_{1}, h}\left(u_{h}, v_{h}\right)=\sum_{\mathrm{P} \in \Omega_{h}} a_{p_{1}, h}^{\mathrm{P}}\left(u_{h}, v_{h}\right)
$$

where each local term $a_{p_{1}, h}^{\mathrm{P}}: V_{h, r}^{p_{2}, p_{1}}(\mathrm{P}) \times V_{h, r}^{p_{2}, p_{1}}(\mathrm{P}) \rightarrow \mathbb{R}$ is a symmetric bilinear form. We set

$$
a_{p_{1}, h}^{P}\left(u_{h}, v_{h}\right)=a_{p_{1}}^{\mathrm{P}}\left(\Pi_{r}^{p_{1}, \mathrm{P}} u_{h}, \Pi_{r}^{p_{1}, \mathrm{P}} v_{h}\right)+S^{\mathrm{P}}\left(u_{h}-\Pi_{r}^{p_{1}, \mathrm{P}} u_{h}, v_{h}-\Pi_{r}^{p_{1}, \mathrm{P}} v_{h}\right),
$$

where the stabilization form $S^{\mathrm{P}}: V_{h, r}^{p_{2}, p_{1}}(\mathrm{P}) \times V_{h, r}^{p_{2}, p_{1}}(\mathrm{P}) \rightarrow \mathbb{R}$ is a symmetric positive definite bilinear form such that

$$
\sigma_{*} a_{p_{1}}^{\mathrm{P}}\left(v_{h}, v_{h}\right) \leq S^{\mathrm{P}}\left(v_{h}, v_{h}\right) \leq \sigma^{*} a_{p_{1}}^{\mathrm{P}}\left(v_{h}, v_{h}\right) \quad \forall v_{h} \in V_{h, r}^{p_{2}, p_{1}}(\mathrm{P}) \text { with } \Pi_{r}^{p_{1}, \mathrm{P}} v_{h}=0,
$$

for two positive constants $\sigma_{*}, \sigma^{*}$ that are independent of $h$ (and P). A possible proof of the validity of 4.10 for the so-called "dofi-dofi" stabilization in the context of arbitrarily regular conforming VEM can be found in Ref. 53 (for the case $p_{1}=2$ see also Refs. 23, 38). The bilinear form $a_{h, \mathrm{P}}(\cdot, \cdot)$ has the two fundamental properties of $r$-consistency and stability, cf. (3.2) and (3.3) 10

\subsection{Discretization of the load term}

We set

$$
\left\langle f_{h}, v_{h}\right\rangle=\sum_{\mathrm{P} \in \Omega_{h}} \int_{\mathrm{P}} f_{h} v_{h} d x d y,
$$

where, for $\mathrm{P} \in \Omega_{h}, f_{h}$ is defined distinguishing two cases

$$
\begin{aligned}
& \text { (a) } \quad f_{h \mid \mathrm{P}}=\Pi_{r-2 p_{1}}^{0, \mathrm{P}} f, \quad \text { if } p_{2}+2 p_{1}-1 \leq r, \\
& \text { (b) } \quad f_{h \mid \mathrm{P}}=\Pi_{r-p_{1}}^{0, \mathrm{P}} f, \quad \text { if } p_{2} \leq r \leq p_{2}+2 p_{1}-2 .
\end{aligned}
$$

Before discussing the two cases (a) and (b) separately, we make the following useful remark. We note that, if $r \geq 2 p_{1}$ and we choose $f_{h}$ as the piecewise polynomial approximation of $f$ on $\Omega_{h}$ given by 4.12 , the right-hand side of 4.11) is fully computable by using only the degrees of freedom (D3), thus by-passing the enhanced approach! 1 
We next discuss the case (a). We choose $f_{h}$ as in 4.12 and we observe that $r-2 p_{1} \geq p_{2}-1$ (that is $r \geq 3 p_{1}-1$, since $p_{2} \geq p_{1}$ ). Thus, using the definition of the $L^{2}$-orthogonal projection, from 4.11, we find that

$$
\left\langle f_{h}, v_{h}\right\rangle=\sum_{\mathrm{P} \in \mathcal{T}_{h}} \int_{\mathrm{P}} \Pi_{r-2 p_{1}}^{0, \mathrm{P}} f v_{h} d x d y=\sum_{\mathrm{P} \in \mathcal{T}_{h}} \int_{\mathrm{P}} \Pi_{r-2 p_{1}}^{0, \mathrm{P}} f \Pi_{p_{1}-1}^{0, \mathrm{P}} v_{h} d x d y
$$

which, in combination with standard approximation results and recalling that $v_{h} \in$ $V_{h, r}^{p_{2}, p_{1}} \subset H_{0}^{p_{1}}(\Omega) \cap H^{p_{2}}(\Omega)$, yields the following estimate:

$$
\left\langle f-f_{h}, v_{h}\right\rangle \lesssim h^{r-p_{1}+1}\left|v_{h}\right|_{p_{1}}|f|_{r-2 p_{1}+1} .
$$

In particular, for $p_{1}=p_{2} \geq 2$ it is enough to choose $r \geq 3 p_{1}-1$ (the case $p_{1}=p_{2}=2$ and $r \geq 5$ has been originally treated in Ref. 35$)$.

We next discuss the case (b). We consider 4.11) with $f_{h}$ defined as in (4.13). We first observe that the computability of 4.11 is ensured by resorting to the local enhancement space that is defined, with an abuse of notation, as follows

$$
\begin{gathered}
V_{h, r}^{p_{2}, p_{1}}(\mathrm{P}):=\left\{v_{h} \in \widetilde{V}_{h, r}^{p_{2}, p_{1}}(\mathrm{P}): \int_{\mathrm{P}} \Pi_{r-p_{1}}^{p_{1}, \mathrm{P}} v_{h} q d \mathbf{x}=\int_{\mathrm{P}} \Pi_{r-p_{1}}^{0, \mathrm{P}} v_{h} q d \mathbf{x}\right. \\
\left.\forall q \in \mathbb{P}_{r-p_{1}} \backslash \mathbb{P}_{r-2 p_{1}}(\mathrm{P})\right\},
\end{gathered}
$$

where we introduced the "extended" virtual element space on element $\mathrm{P}$ defined by $\widetilde{V}_{h, r}^{p_{2}, p_{1}}(\mathrm{P})=\left\{v_{h} \in H^{p_{2}}(\mathrm{P}): \Delta^{p_{2}} v_{h} \in \mathbb{P}_{r-p_{1}}(\mathrm{P}), \partial_{n}^{i} v_{h} \in \mathbb{P}_{r-i}(e), i=0, \ldots, p_{2}-\right.$ $1 \forall e \in \partial \mathrm{P}\}$. Due to the bound on $r$, we have $r-2 p_{1} \leq p_{2}-2$, and $\mathbb{P}_{r-p_{1}} \backslash \mathbb{P}_{r-2 p_{1}}(\mathrm{P})$ denotes the space of homogeneous polynomials of degree $\ell$ with $r-2 p_{1}+1 \leq$ $\ell \leq r-p_{1}$ defined on P. Similarly to the previous case, using the definition of the $L^{2}$-orthogonal projection we find that

$$
\left\langle f_{h}, v_{h}\right\rangle=\sum_{\mathrm{P} \in \mathcal{T}_{h}} \int_{\mathrm{P}} \Pi_{r-p_{1}}^{0, \mathrm{P}} f v_{h} d x d y=\sum_{\mathrm{P} \in \mathcal{T}_{h}} \int_{\mathrm{P}} \Pi_{r-p_{1}}^{0, \mathrm{P}} f \Pi_{0}^{0, \mathrm{P}} v_{h} d x d y
$$

which yields

$$
\left\langle f-f_{h}, v_{h}\right\rangle \lesssim h^{r-p_{1}+1}\left|v_{h}\right|_{p_{1}}|f|_{r-p_{1}+1} .
$$

We underline that in this paper we have decided to employ the enhancement approach as it represents a powerful and unifying framework to treat the approximation of the right-hand side. It is worth mentioning that in the case $p_{2}=p_{1}=2$ and $r-2 p_{1} \leq 0$ the enhancement approach can be avoided by employing ad-hoc arguments ${ }^{35}$ For arbitrary values of $p_{1}$ and $p_{2}$, the use of the enhancement approach might be avoided using arguments similar to those employed in Ref. 52 .

\subsection{Error analysis}

In this section, we recall some convergence results for the approximation of (2.1). In particular, employing Theorem 3.1 together with standard approximation results 
(see, e.g. Ref. 20) and the approximation properties of the right-hand side contained in Sec. 4.5 , the following convergence result in the energy norm holds 10

Theorem 4.4. Let $u \in H_{0}^{p_{1}}(\Omega) \cap H^{r+1}(\Omega)$ be the solution of the polyharmonic problem 2.1 and let $u_{h} \in V_{h, r}^{p_{2}, p_{1}}$ be the solution of the virtual element method (3.1). Assume $f \in H^{s}(\Omega)$ where $s=r-\left(p_{1}-1\right)$ if $p_{2} \leq r \leq p_{2}+2 p_{1}-2$ and $s=r-\left(2 p_{1}-1\right)$ if $r \geq p_{2}+2 p_{1}-1$. Then, it holds that

$$
\left\|u-u_{h}\right\|_{\vee} \leq C h^{r-\left(p_{1}-1\right)}\left(|u|_{r+1}+|f|_{s}\right) .
$$

Proof. The assertion follows from the abstract convergence result stated in Theorem 3.1 and estimating each term on the right-hand side of (3.4) separately based on employing classical interpolation bounds and the approximation properties of the right-hand side discussed in Sec. 4.5.

We remark that in this paper we focus on error bounds in the energy norm; convergence estimates in lower-order norms can be established provided that classical duality arguments can be used and that the polynomial approximation order $r$ is sufficient large $10 \mid 41$

\section{Numerics}

We investigate the behavior of the two-dimensional, highly-regular, conforming virtual element approximations that we introduced in the previous sections when applied to the numerical resolution of the Poisson $\left(p_{1}=1\right)$ and biharmonic $\left(p_{1}=2\right)$ equations.

According to the notation introduced in Sec. 3, we recall that the finitedimensional virtual element space $V_{h, r}^{p_{2}, p_{1}}$ is a subspace of $H_{0}^{p_{2}}(\Omega)$. Moreover, the local Virtual Element space $V_{h, r}^{p_{2}, p_{1}}(\mathrm{P})$, i.e. the restriction of $V_{h, r}^{p_{2}, p_{1}}$ to any element $\mathrm{P} \in \Omega_{h}$, is a finite-dimensional subspace of $H^{p_{2}}(\mathrm{P})$ containing the space of polynomials of degree up to $r$ defined on $\mathrm{P}$.

Throughout the section, the computational domain is the unit square, the loading term $f$ is set up in accordance with the exact solution

$$
u(x, y)=(1-x)^{2} x^{2}(1-y)^{2} y^{2},
$$

and the boundary conditions are chosen accordingly.

We consider four different mesh families: quadrilateral meshes QUAD, triangular meshes TRI, central Voronoi tessellations CVT and hexagonal meshes HEX. An example of a mesh of each family is shown in Fig. 1 the corresponding number of elements of the refined meshes is shown in Table 1

To illustrate the two stabilization strategies that we are going to test in practice, we rewrite Eq. 4.9) in matrix form, i.e.

$$
A_{P}=M_{P}+S_{P}
$$




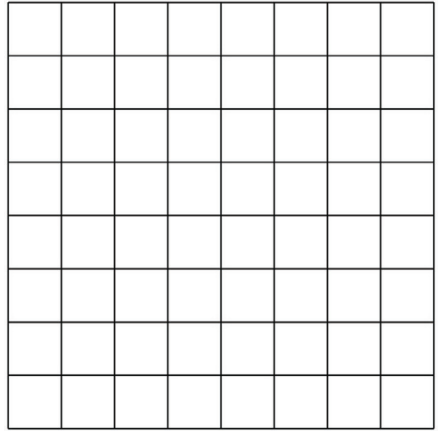

(a) Quadrilateral (QUAD) mesh

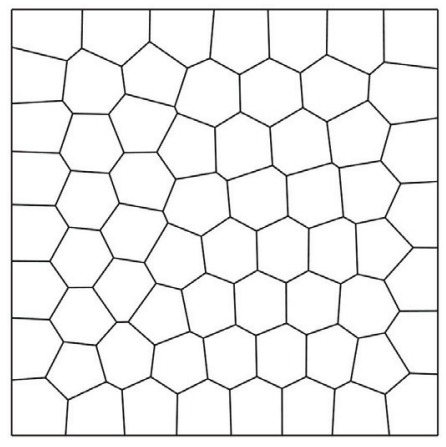

(c) CVT (CVT) mesh

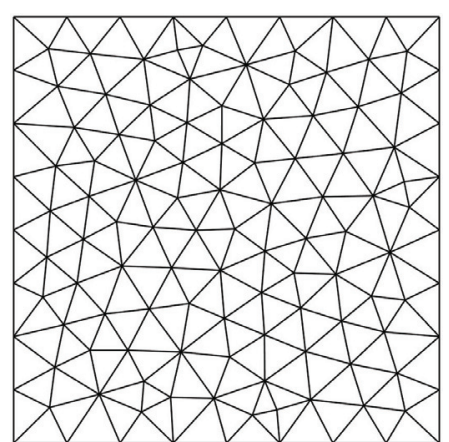

(b) Triangular (TRI) mesh

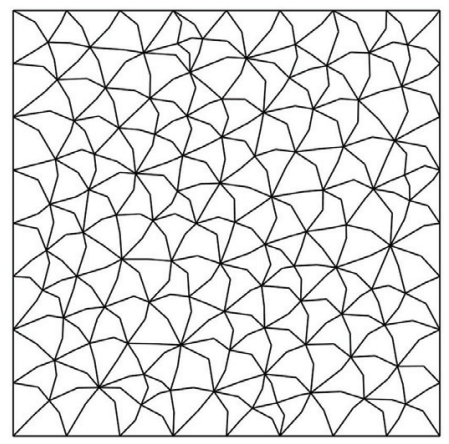

(d) Exagonal (HEX) mesh

Fig. 1. Examples of polygonal meshes used in the numerical tests of Sec. 5 a quadrilateral (QUAD), triangular (TRI), central Voronoi CVT, and hexagonal (HEX) mesh.

Table 1. Number of elements of the sequences of meshes versus the inverse of the mesh sixe $h$.

\begin{tabular}{lccccc}
\hline $1 / h$ & 8 & 16 & 32 & 64 & 128 \\
\hline QUAD & 64 & 256 & 1024 & 4096 & 16,384 \\
TRI & 212 & 870 & 3486 & 14,080 & 56,932 \\
CVT & 64 & 256 & 1024 & 4096 & 16,384 \\
HEX & 212 & 870 & 3486 & 14,080 & 56,932 \\
\hline
\end{tabular}

where $A_{P}$ is the elemental stiffness matrix, $M_{P}$ is the consistency matrix associated with $a_{p_{1}}^{\mathrm{P}}\left(\Pi_{r}^{p_{1}, \mathrm{P}} u_{h}, \Pi_{r}^{p_{1}, \mathrm{P}} v_{h}\right)$ and $\mathrm{S} \mathrm{P}$ is the stabilization matrix associated with $S^{\mathrm{P}}\left(u_{h}-\Pi_{r}^{p_{1}, \mathrm{P}} u_{h}, v_{h}-\Pi_{r}^{p_{1}, \mathrm{P}} v_{h}\right)$. The matrix $\mathrm{S}_{\mathrm{P}}$ has the following structure:

$$
\mathrm{S}_{\mathrm{P}}=\alpha_{\text {stab }}(\mathrm{I}-\mathrm{DQ})^{T} \mathrm{U}(\mathrm{I}-\mathrm{DQ}),
$$

where $\alpha_{\text {stab }}$ is a scalar factor ensuring that matrices $\mathrm{M}_{\mathrm{P}}$ and $\mathrm{S}_{\mathrm{P}}$ have the same scaling with respect to $h$ and $I$ is the identity matrix. Moreover, in (5.2), Q is the matrix representation of the polynomial projection operator $\Pi_{r}^{p_{1}, \mathrm{P}}$, i.e. the matrix whose 
columns are the coefficients of the projection of the canonical basis functions with respect to a given polynomial basis of $\mathbb{P}_{r}(\mathrm{P})$; $\mathrm{D}$ is the matrix collecting the degrees of freedom of such polynomial basis on its column; and, finally, $U$ is a suitable matrix that allows us to change the Virtual Element stabilization. In particular, we consider the following two possible choices of $U$ given in

- $\mathrm{U}=\mathrm{I}$, which is sometimes called in the virtual element jargon the "dofi-dofi stabilization";

- $\mathrm{U}=\mathrm{D}^{\perp}=\mathrm{I}-\mathrm{D}\left(\mathrm{D}^{T} \mathrm{D}\right)^{-1} \mathrm{D}^{T}$.

In the second choice above, we use the symbol $\mathrm{D}^{\perp}$ to outline the fact that this matrix operator is the orthogonal projector onto the complement of the vector space spanned by the columns of D (so, we can call it the "D-perp stabilization"). Since $\mathrm{D}$ is a maximum rank matrix by definition, the square matrix $\mathrm{D}^{T} \mathrm{D}$ is a square nonsingular matrix, and thus the matrix $U$ is well-defined. Other possible stabilization strategies for the VEM can be designed according to Refs. 45 and 56.

In the solution of the Poisson equation ( i.e. $p_{1}=1$, cf. Sec. 5.1) we take $\alpha_{\text {stab }}=\operatorname{Trace}\left(\mathrm{M}_{\mathrm{P}}\right) / N^{\text {dofs }}$, where $N^{\text {dofs }}$ is the number of rows (or columns) of matrix $\mathrm{M}_{\mathrm{P}}$, i.e. the local number of degrees of freedom. In the solution of the biharmonic equation (i.e. $p_{1}=2$, cf. Sec. 5.2), the factor $\alpha$ must scale as $h^{-2}$, and the choice is not unique. To our purpose, we consider three different choices of this parameter, which we will detail in Sec. 5.2.

In each test case, we compare the condition number of the stiffness matrix and the accuracy of the resulting approximation by measuring the error in the energy norm and in the $L^{2}$-norm (Poisson equation) and in the energy norm and in the $L^{\infty}$-norm (biharmonic equation). We point out that the experimental estimation of the condition number of the global stiffness matrix A has been obtained by exploiting the analogies between the Lanczos technique and the Conjugate Gradient method. Indeed, within the Conjugate Gradient algorithm we can build a suitable tridiagonal matrix whose extreme eigenvalues converge to the extreme eigenvalues of A, see Secs. 9.3 and 10.2 in Ref. 49 for more details. The approximation error is evaluated by computing $e_{h}=u-\Pi_{r}^{p_{1}} u_{h}$, and its energy norm is provided by $\left(a_{p_{1}, h}\left(e_{h}, e_{h}\right)\right)^{1 / 2}$. Finally, it is informative to say that we carried out all the tests of this section by using our in-house $C^{++}$and MATLAB 59 implementations.

\subsection{Poisson equation}

We recall that, for fixed $p_{2} \geq p_{1}, k=p_{2}-1$ denotes the regularity of the global virtual element space, and that $r$ denotes the degree of the polynomials contained in each elemental approximation space. We carried out the calculations corresponding to the two following test cases (TCs):

- TC1: $(k=0, r=2),(k=1, r=2)$;

- TC2: $(k=0, r=3),(k=1, r=3),(k=2, r=3)$. 

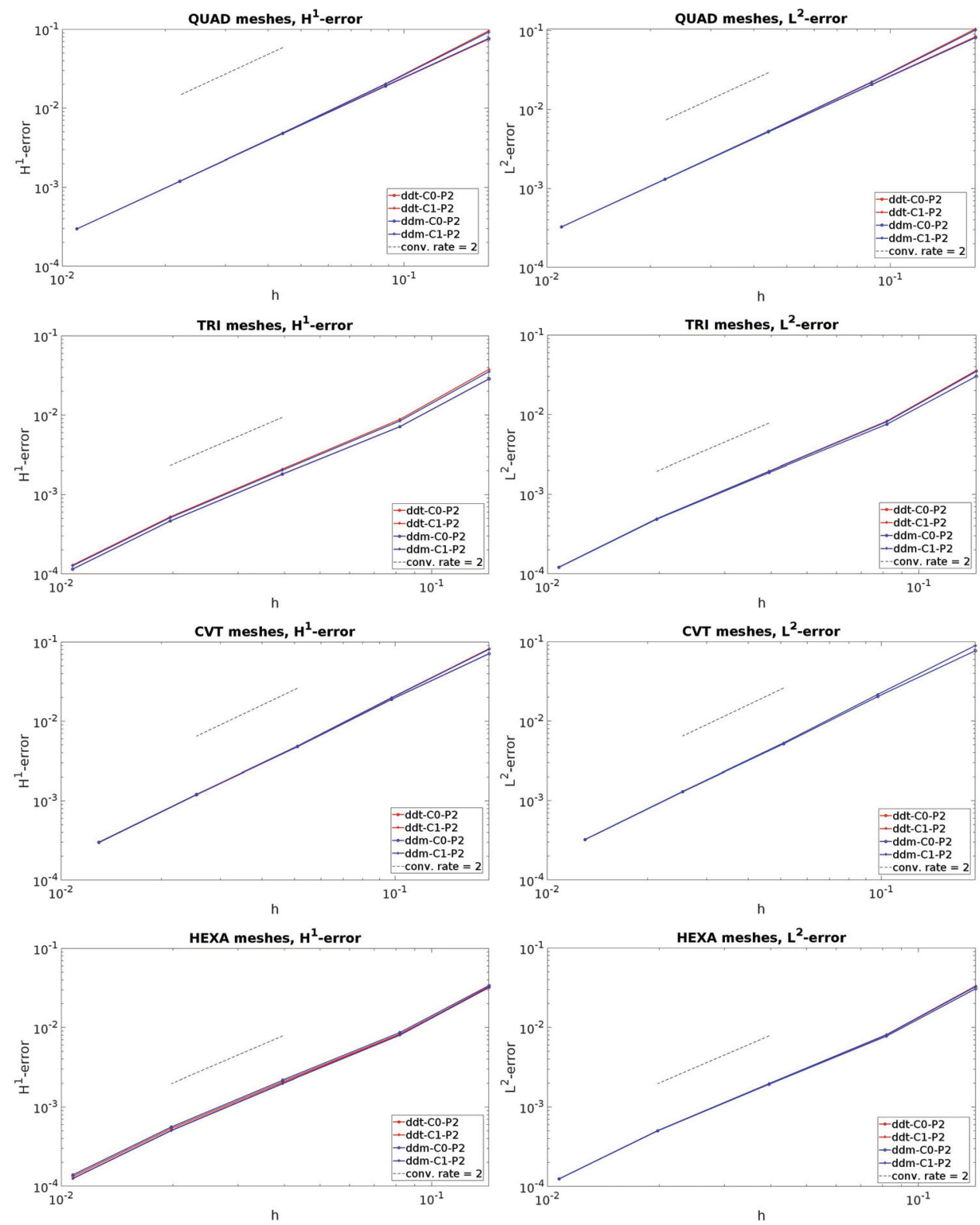

Fig. 2. (Color online) Poisson equation, test case TC1. Plots of the error curves versus the mesh size parameter $h$ for the discretization using the (reduced) virtual element space of Sec. 4.3 with $p_{1}=1, p_{2}=1,2, r=2$ on different polygonal mesh families and stabilization terms (ddt and ddm in the legend refer to $\mathrm{U}=\mathrm{I}$ and $\mathrm{U}=\mathrm{D}^{\perp}$ in $[5.2$, respectively). The errors are measured using the energy norm (left) and the $L^{2}$-norm (right), and are expected to scale proportionally to $h^{2}$. 

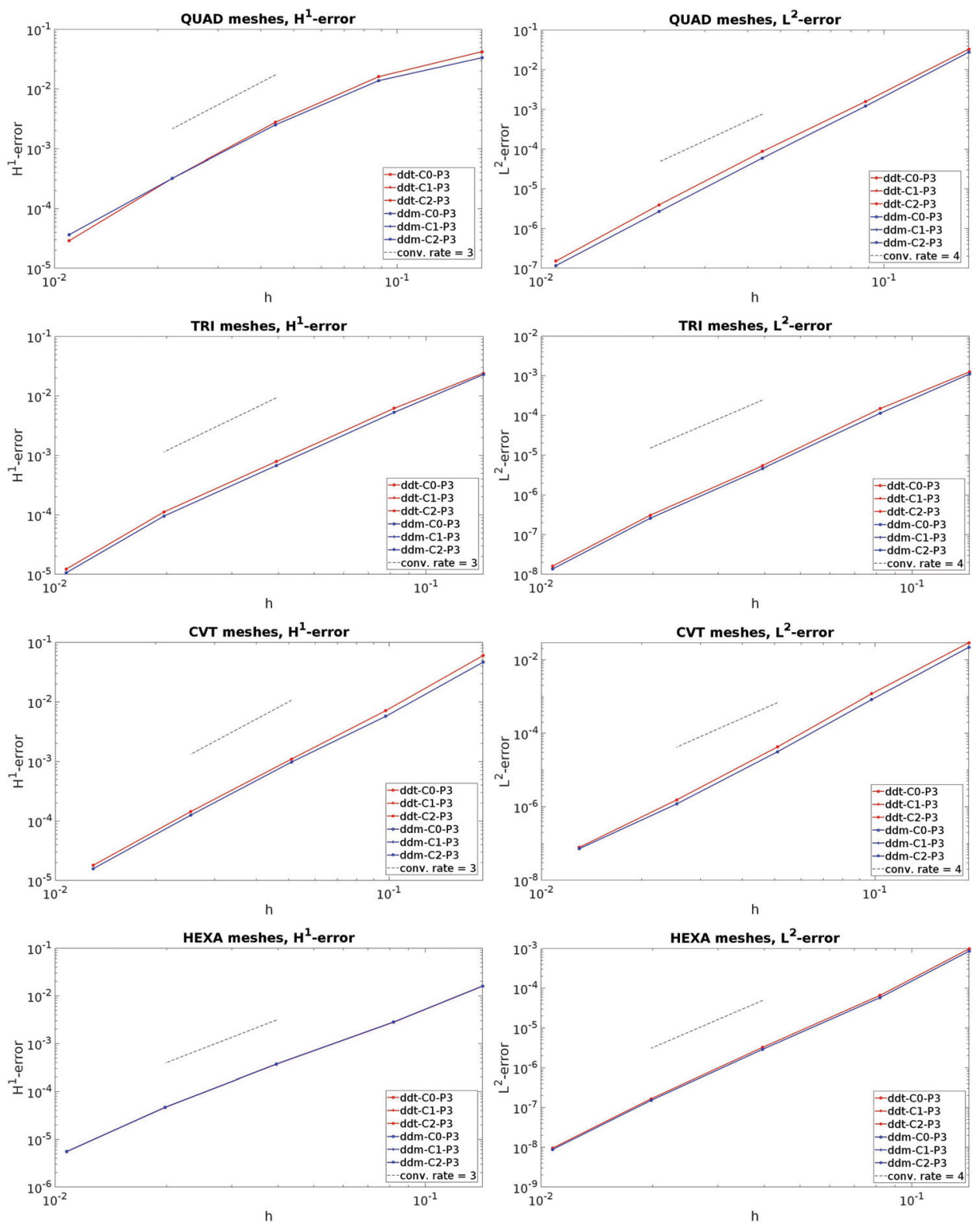

Fig. 3. (Color online) Poisson equation, test case TC2. Plots of the error curves versus the mesh size parameter $h$ for the discretization using the (reduced) virtual element space of Sec. 4.3 with $p_{1}=1, p_{2}=1,2,3, r=3$ on different polygonal mesh families and stabilization terms (ddt and $\mathrm{ddm}$ in the legend refer to $\mathrm{U}=\mathrm{I}$ and $\mathrm{U}=\mathrm{D}^{\perp}$ in $(5.2)$, respectively). The errors are measured using the energy norm (left) and the $L^{2}$-norm (right), and are expected to scale proportionally to $h^{3}$ and $h^{4}$, respectively. 
For both test cases, we consider the four different mesh families shown in Fig. 1 and the two possible choices of the stabilizing bilinear form discussed above, i.e. by choosing $U=I$ and $U=D^{\perp}$ in 5.2 . In every calculation, we measure the error in the energy norm $\left(H^{1}\right.$-norm) and in the $L^{2}$-norm, and we evaluate the condition number of the stiffness matrix. The plots of the error curves versus $h$ (loglog scale) are shown in Figs. 2 and 3 . The computed condition numbers are reported in Tables 2 and 3 . We observe that the two different stabilizations seem to provide comparable results concerning the condition numbers, which is exhibiting the expected growth $O\left(h^{-2}\right)$, although the choice $\mathrm{U}=\mathrm{D}^{\perp}$ seems to provide lower condition numbers for the VEM with higher regularity.

The behavior of the error curves is also very similar for all these variants of the VEM, the error curves being very closed in almost every plot and overlapping to the point that they cannot easily be distinguished. Optimal convergence rates are seen in every plot. We recall that the error in energy norm is expected to decrease proportionally to $h^{r}$ for $h \rightarrow 0$ for all values of the polynomial order (order of accuracy of the method) $r$ here considered. Instead, the error in the $L^{2}$-norm is expected to reduce as $h^{2}$ for $r=2$ and $h^{4}$ for $r=3$, as we do not

Table 2. Poisson equation. Comparison of the computed condition number estimates obtained with the different stabilization strategies, different regularity $k=0,1$ and polynomial order $r=2$.

\begin{tabular}{|c|c|c|c|c|}
\hline \multirow[b]{2}{*}{$1 / h$} & \multicolumn{2}{|c|}{$C^{0}-\mathbb{P}_{2}$} & \multicolumn{2}{|c|}{$C^{1}-\mathbb{P}_{2}$} \\
\hline & $\mathrm{U}=\mathrm{I}$ & $\mathrm{U}=\mathrm{D}^{\perp}$ & $\mathrm{U}=\mathrm{I}$ & $\mathrm{U}=\mathrm{D}^{\perp}$ \\
\hline & \multicolumn{4}{|c|}{ QUAD meshes } \\
\hline 8 & $1.24 \mathrm{e}+3$ & $4.55 \mathrm{e}+2$ & $2.38 \mathrm{e}+3$ & $3.04 \mathrm{e}+2$ \\
\hline 16 & $4.99 \mathrm{e}+3$ & $1.83 e+3$ & $1.04 \mathrm{e}+4$ & $1.29 \mathrm{e}+3$ \\
\hline 32 & $2.00 \mathrm{e}+4$ & $7.35 \mathrm{e}+3$ & $4.31 \mathrm{e}+4$ & $5.28 \mathrm{e}+3$ \\
\hline 64 & $8.01 \mathrm{e}+4$ & $2.94 \mathrm{e}+4$ & $1.75 \mathrm{e}+5$ & $2.13 \mathrm{e}+4$ \\
\hline \multirow[t]{2}{*}{128} & $3.21 \mathrm{e}+5$ & $1.18 \mathrm{e}+5$ & $7.05 \mathrm{e}+5$ & $8.59 \mathrm{e}+4$ \\
\hline & \multicolumn{4}{|c|}{ TRI meshes } \\
\hline 8 & $9.97 \mathrm{e}+3$ & $1.37 \mathrm{e}+3$ & $1.59 \mathrm{e}+4$ & $1.09 \mathrm{e}+3$ \\
\hline 16 & $4.27 \mathrm{e}+4$ & $5.97 \mathrm{e}+3$ & $7.49 \mathrm{e}+4$ & $5.14 \mathrm{e}+3$ \\
\hline 32 & $1.69 \mathrm{e}+5$ & $2.37 \mathrm{e}+4$ & $3.27 \mathrm{e}+5$ & $1.99 \mathrm{e}+4$ \\
\hline 64 & $6.80 \mathrm{e}+5$ & $9.45 \mathrm{e}+4$ & $1.31 \mathrm{e}+6$ & $8.26 \mathrm{e}+4$ \\
\hline \multirow[t]{2}{*}{128} & $2.79 \mathrm{e}+6$ & $3.89 \mathrm{e}+5$ & $5.50 \mathrm{e}+6$ & $3.34 \mathrm{e}+5$ \\
\hline & \multicolumn{4}{|c|}{ CVT meshes } \\
\hline 8 & $1.53 \mathrm{e}+3$ & $6.37 \mathrm{e}+2$ & $1.95 \mathrm{e}+3$ & $3.69 \mathrm{e}+2$ \\
\hline 16 & $6.32 \mathrm{e}+3$ & $2.62 \mathrm{e}+3$ & $8.97 \mathrm{e}+3$ & $1.51 \mathrm{e}+3$ \\
\hline 32 & $2.70 \mathrm{e}+4$ & $1.04 \mathrm{e}+4$ & $3.97 \mathrm{e}+4$ & $6.22 \mathrm{e}+3$ \\
\hline 64 & $1.02 \mathrm{e}+5$ & $4.15 \mathrm{e}+4$ & $1.65 \mathrm{e}+5$ & $2.47 \mathrm{e}+4$ \\
\hline \multirow[t]{2}{*}{128} & $4.14 \mathrm{e}+5$ & $1.64 \mathrm{e}+5$ & $6.62 \mathrm{e}+5$ & $9.82 \mathrm{e}+4$ \\
\hline & \multicolumn{4}{|c|}{ HEX meshes } \\
\hline 8 & $8.88 \mathrm{e}+3$ & $2.23 \mathrm{e}+3$ & $1.10 \mathrm{e}+4$ & $1.26 \mathrm{e}+3$ \\
\hline 16 & $4.08 \mathrm{e}+4$ & $9.72 \mathrm{e}+3$ & $5.44 \mathrm{e}+4$ & $3.70 \mathrm{e}+3$ \\
\hline 32 & $2.22 \mathrm{e}+5$ & $3.88 \mathrm{e}+4$ & $2.42 \mathrm{e}+5$ & $2.21 \mathrm{e}+4$ \\
\hline 64 & $8.79 \mathrm{e}+5$ & $1.64 \mathrm{e}+5$ & $1.23 \mathrm{e}+6$ & $9.02 \mathrm{e}+4$ \\
\hline 128 & $5.57 \mathrm{e}+6$ & $6.65 \mathrm{e}+5$ & $5.01 \mathrm{e}+6$ & $3.73 \mathrm{e}+5$ \\
\hline
\end{tabular}


Table 3. Poisson equation. Comparison of the condition number estimates obtained with the different stabilization strategies, different regularity $k=0,1,2$ and polynomial order $r=3$. The acronym "n.a." stands for "not available" since the resulting stiffness matrix was too badly-conditioned to estimate the condition number.

\begin{tabular}{|c|c|c|c|c|c|c|}
\hline \multirow[b]{2}{*}{$1 / h$} & \multicolumn{2}{|c|}{$C^{0}-\mathbb{P}_{3}$} & \multicolumn{2}{|c|}{$C^{1}-\mathbb{P}_{3}$} & \multicolumn{2}{|c|}{$C^{2}-\mathbb{P}_{3}$} \\
\hline & $\mathrm{U}=\mathrm{I}$ & $\mathrm{U}=\mathrm{D}^{\perp}$ & $\mathrm{U}=\mathrm{I}$ & $\mathrm{U}=\mathrm{D}^{\perp}$ & $\mathrm{U}=\mathrm{I}$ & $\mathrm{U}=\mathrm{D}^{\perp}$ \\
\hline & \multicolumn{6}{|c|}{ QUAD meshes } \\
\hline 8 & $3.74 \mathrm{e}+4$ & $1.32 \mathrm{e}+6$ & $7.67 \mathrm{e}+6$ & $6.70 \mathrm{e}+3$ & $8.41 \mathrm{e}+6$ & $3.25 \mathrm{e}+3$ \\
\hline 16 & $1.49 \mathrm{e}+5$ & $5.26 \mathrm{e}+6$ & $2.90 \mathrm{e}+7$ & $2.50 \mathrm{e}+4$ & $5.52 \mathrm{e}+7$ & $1.98 \mathrm{e}+4$ \\
\hline 32 & $5.96 \mathrm{e}+5$ & $2.10 \mathrm{e}+7$ & $1.16 \mathrm{e}+8$ & $9.92 \mathrm{e}+4$ & $2.50 \mathrm{e}+8$ & $8.93 \mathrm{e}+4$ \\
\hline 64 & $2.38 \mathrm{e}+6$ & $8.41 \mathrm{e}+7$ & $4.66 \mathrm{e}+8$ & $3.97 \mathrm{e}+5$ & $1.05 \mathrm{e}+9$ & $3.72 \mathrm{e}+5$ \\
\hline \multirow[t]{2}{*}{128} & $9.53 \mathrm{e}+6$ & $3.36 \mathrm{e}+8$ & $1.87 \mathrm{e}+9$ & $1.59 \mathrm{e}+6$ & $4.30 \mathrm{e}+9$ & $1.52 \mathrm{e}+6$ \\
\hline & \multicolumn{6}{|c|}{ TRI meshes } \\
\hline 8 & $4.88 \mathrm{e}+5$ & $1.26 \mathrm{e}+8$ & $2.74 \mathrm{e}+9$ & $1.72 \mathrm{e}+5$ & $5.91 \mathrm{e}+9$ & $1.05 \mathrm{e}+5$ \\
\hline 16 & $2.43 \mathrm{e}+6$ & $6.87 \mathrm{e}+8$ & $1.56 \mathrm{e}+10$ & $8.01 \mathrm{e}+5$ & $5.39 \mathrm{e}+10$ & $6.53 \mathrm{e}+5$ \\
\hline 32 & $1.15 \mathrm{e}+7$ & $3.73 e+9$ & $9.67 \mathrm{e}+10$ & $3.75 \mathrm{e}+6$ & $3.60 \mathrm{e}+11$ & $3.74 \mathrm{e}+6$ \\
\hline 64 & $4.14 \mathrm{e}+7$ & $1.25 \mathrm{e}+10$ & n.a. & $1.35 \mathrm{e}+7$ & n.a. & $1.27 \mathrm{e}+7$ \\
\hline \multirow[t]{2}{*}{128} & $2.15 \mathrm{e}+8$ & $7.39 \mathrm{e}+10$ & n.a. & $6.82 \mathrm{e}+7$ & n.a. & $6.82 \mathrm{e}+7$ \\
\hline & \multicolumn{6}{|c|}{ CVT meshes } \\
\hline 8 & $8.79 \mathrm{e}+4$ & $4.98 \mathrm{e}+6$ & $3.31 e+7$ & $2.14 \mathrm{e}+4$ & $3.83 \mathrm{e}+7$ & $1.34 \mathrm{e}+4$ \\
\hline 16 & $3.91 \mathrm{e}+5$ & $2.22 \mathrm{e}+7$ & $1.95 \mathrm{e}+8$ & $1.05 \mathrm{e}+5$ & $2.91 \mathrm{e}+8$ & $8.55 \mathrm{e}+4$ \\
\hline 32 & $1.56 \mathrm{e}+6$ & $8.23 \mathrm{e}+7$ & $8.29 \mathrm{e}+8$ & $3.65 \mathrm{e}+5$ & $1.50 \mathrm{e}+9$ & $3.27 \mathrm{e}+5$ \\
\hline 64 & $8.03 e+6$ & $3.45 e+8$ & $7.62 \mathrm{e}+9$ & $2.20 \mathrm{e}+6$ & $1.51 \mathrm{e}+10$ & $2.08 \mathrm{e}+6$ \\
\hline \multirow[t]{2}{*}{128} & $2.69 \mathrm{e}+7$ & n.a. & n.a. & $7.85 \mathrm{e}+6$ & $5.00 \mathrm{e}+10$ & $7.55 \mathrm{e}+6$ \\
\hline & \multicolumn{6}{|c|}{ HEX meshes } \\
\hline 8 & $8.94 \mathrm{e}+5$ & $1.87 \mathrm{e}+8$ & $3.07 \mathrm{e}+8$ & $1.24 \mathrm{e}+5$ & $9.13 \mathrm{e}+8$ & $9.65 \mathrm{e}+4$ \\
\hline 16 & $4.82 \mathrm{e}+6$ & $1.50 \mathrm{e}+9$ & $1.80 \mathrm{e}+9$ & $6.50 \mathrm{e}+5$ & $6.37 \mathrm{e}+9$ & $5.75 \mathrm{e}+5$ \\
\hline 32 & $2.56 \mathrm{e}+7$ & $8.47 \mathrm{e}+9$ & $1.87 \mathrm{e}+10$ & $3.55 \mathrm{e}+6$ & $1.65 \mathrm{e}+10$ & $3.33 \mathrm{e}+6$ \\
\hline 64 & $8.98 \mathrm{e}+7$ & $1.99 \mathrm{e}+10$ & $8.91 e+10$ & $1.57 \mathrm{e}+7$ & $2.58 \mathrm{e}+11$ & $1.51 \mathrm{e}+7$ \\
\hline 128 & n.a. & $1.05 \mathrm{e}+11$ & $3.24 \mathrm{e}+11$ & $5.67 \mathrm{e}+7$ & n.a. & $5.53 \mathrm{e}+7$ \\
\hline
\end{tabular}

adopted the modified, e.g. "enhanced", version of the VEM, 1 which makes a better approximation to the solution possible for the low order case $r=2$. This loss of an order of convergence is a well-known phenomenon and has been discussed in a previous paper.26

Table 4. Biharmonic equation, QUAD meshes. Comparison of the condition numbers obtained with the different stabilization strategies.

\begin{tabular}{r|c|cc|cc}
\hline & & \multicolumn{2}{|c|}{$\alpha_{\text {stab }}=\operatorname{Trace}\left(\mathrm{M}_{\mathrm{P}}\right) / 3$} & \multicolumn{2}{c}{$\alpha_{\text {stab }}=1 /|\mathrm{P}|$} \\
$1 / h$ & $N^{\text {dofs }}$ & $\mathrm{U}=\mathrm{I}$ & $\mathrm{U}=\mathrm{D}^{\perp}$ & $\mathrm{U}=\mathrm{I}$ & $\mathrm{U}=\mathrm{D}^{\perp}$ \\
\hline 8 & 243 & $5.77 \mathrm{e}+2$ & $1.93 \mathrm{e}+2$ & $3.41 \mathrm{e}+2$ & $2.08 \mathrm{e}+3$ \\
16 & 867 & $7.68 \mathrm{e}+3$ & $2.47 \mathrm{e}+3$ & $3.96 \mathrm{e}+3$ & $2.28 \mathrm{e}+3$ \\
32 & 3267 & $1.15 \mathrm{e}+5$ & $3.65 \mathrm{e}+4$ & $5.50 \mathrm{e}+4$ & $3.09 \mathrm{e}+4$ \\
64 & 12,675 & $1.81 \mathrm{e}+6$ & $5.72 \mathrm{e}+5$ & $8.42 \mathrm{e}+5$ & $4.69 \mathrm{e}+5$ \\
128 & 49,923 & $2.88 \mathrm{e}+7$ & $9.11 \mathrm{e}+6$ & $1.33 \mathrm{e}+7$ & $7.40 \mathrm{e}+6$ \\
\hline
\end{tabular}



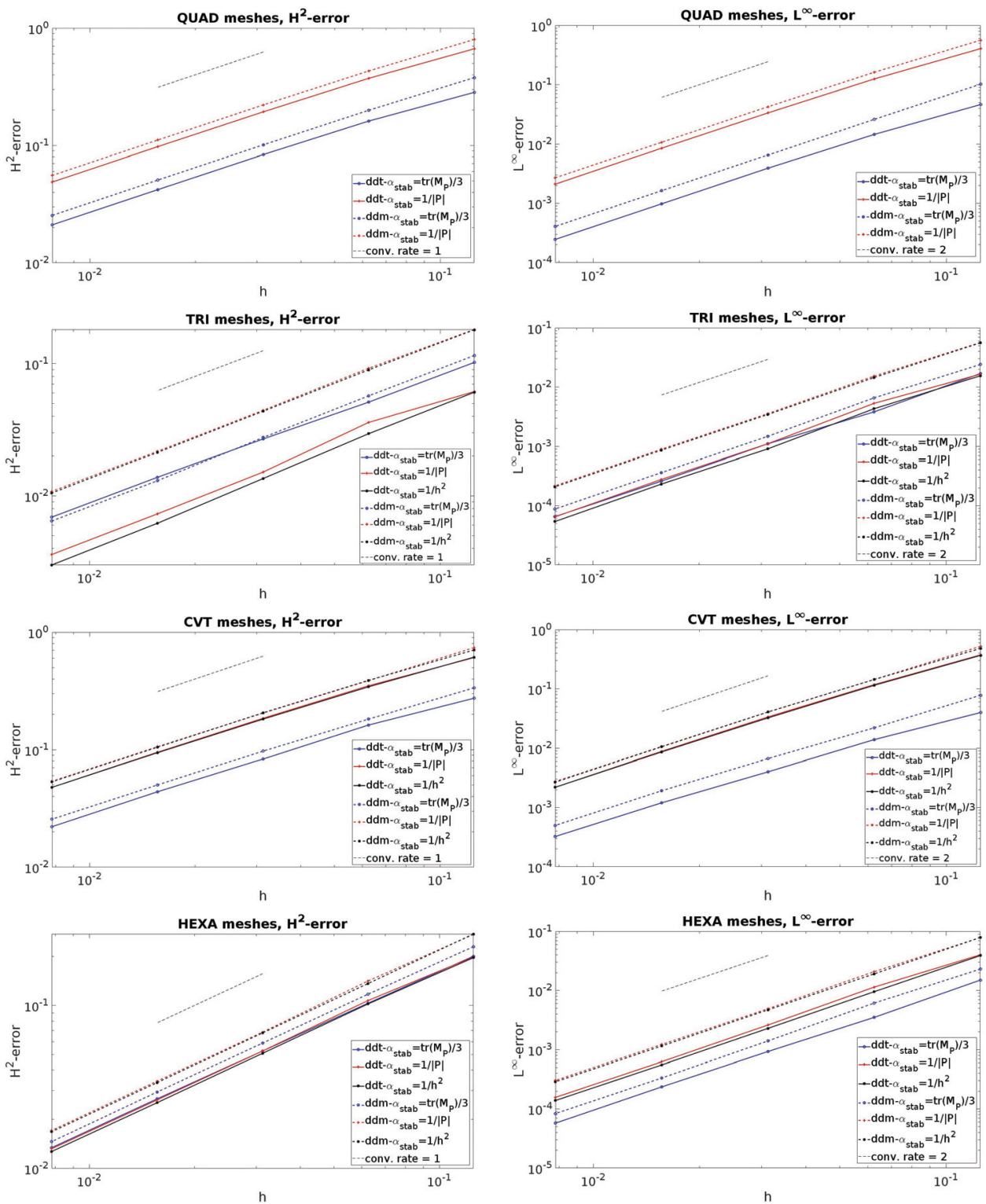

Fig. 4. (Color online) Biharmonic equation. Plots of the error curves versus the mesh size $h$ on different polygonal mesh families and stabilization terms. (ddm and ddt in the legend refer to $U=I$ and $\mathrm{U}=\mathrm{D}^{\perp}$ in $\sqrt{5.2}$, respectively) The errors are measured using the $H^{2}$-norm (left panels) and the $L^{\infty}$-norm (right panel), and are expected to scale proportionally to $h$ and $h^{2}$, respectively. 


\subsection{Biharmonic equation}

In this section, we solve the two-dimensional biharmonic equation using the conforming $C^{1}$ virtual element approximation corresponding to the parameters choice $p_{2}=p_{1}=r=2$. We consider the two stabilization strategies for $\mathrm{U}=\mathrm{I}$ and $\mathrm{U}=\mathrm{D}^{\perp}$ and the three possible choices of the parameter $\alpha_{\text {stab }}$ that are given by

- $\alpha_{\text {stab }}=\operatorname{Trace}\left(\mathrm{M}_{\mathrm{P}}\right) / 3$;

- $\alpha_{\text {stab }}=1 /|\mathrm{P}|$;

- $\alpha_{\text {stab }}=1 / h^{2}$.

In Table 4 and Fig. 4 (first row from top), we report the computed condition number estimates and the error curves for the family of quadrilateral meshes (QUAD). Note that $|\mathrm{P}|=h^{2}$, so the stabilizations for these two corresponding choices of $\alpha_{\text {stab }}$ coincide and only one set of results is shown. All the stabilizations considered seem comparable in terms of the condition number of the resulting linear system of equations, exhibiting the expected growth $O\left(h^{-4}\right)$. Also they seem comparable in terms of accuracy, since the convergence rate is the same ( 1 for the $H^{2}$ norm and 2 for the $L^{\infty}$ norm). However, the stabilization with $\mathrm{U}=\mathrm{I}$ and $\alpha_{\text {stab }}=\operatorname{Trace}\left(\mathrm{M}_{\mathrm{P}}\right) / 3$ yields the best accuracy.

In Table 5 and Fig. 4 (second row), we report the results obtained on triangular meshes (TRI). Again all stabilizations considered exhibit the same behavior in terms of condition number growth and convergence rate, when refining the mesh. Differently from the case of quadrilateral meshes, the best accuracy is obtained using the stabilization with $\mathrm{U}=\mathrm{I}$ and $\alpha_{\text {stab }}=1 / h^{2}$. Tables 6, 7 and Fig. 4 (third and fourth rows) report then the results obtained on CVT and HEX meshes. In these

Table 5. Biharmonic equation, TRI meshes. Comparison of the condition number estimates obtained with the different stabilization strategies.

\begin{tabular}{r|c|cc|cc|cr}
\hline \multirow{2}{*}{} & \multirow{2}{*}{$N^{\text {dofs }}$} & \multicolumn{2}{|c|}{$\alpha_{\text {stab }}=\operatorname{Trace}\left(\mathrm{M}_{\mathrm{P}}\right) / 3$} & \multicolumn{2}{c|}{$\alpha_{\text {stab }}=1 /|\mathrm{P}|$} & \multicolumn{2}{c}{$\alpha_{\text {stab }}=1 / h^{2}$} \\
$1 / h$ & $\mathrm{U}=\mathrm{I}$ & $\mathrm{U}=\mathrm{D}^{\perp}$ & $\mathrm{U}=\mathrm{I}$ & $\mathrm{U}=\mathrm{D}^{\perp}$ & $\mathrm{U}=\mathrm{I}$ & $\mathrm{U}=\mathrm{D}^{\perp}$ \\
\hline 8 & 369 & $2.34 \mathrm{e}+3$ & $6.43 \mathrm{e}+2$ & $1.46 \mathrm{e}+3$ & $5.50 \mathrm{e}+2$ & $9.68 \mathrm{e}+2$ & $4.96 \mathrm{e}+2$ \\
16 & 1404 & $5.27 \mathrm{e}+4$ & $1.31 \mathrm{e}+4$ & $3.16 \mathrm{e}+4$ & $1.08 \mathrm{e}+4$ & $1.90 \mathrm{e}+4$ & $9.52 \mathrm{e}+3$ \\
32 & 5424 & $9.46 \mathrm{e}+5$ & $2.01 \mathrm{e}+5$ & $5.96 \mathrm{e}+5$ & $1.71 \mathrm{e}+5$ & $3.10 \mathrm{e}+5$ & $1.48 \mathrm{e}+5$ \\
64 & 21,507 & $1.39 \mathrm{e}+7$ & $3.35 \mathrm{e}+6$ & $8.76 \mathrm{e}+6$ & $2.75 \mathrm{e}+6$ & $4.92 \mathrm{e}+6$ & $2.40 \mathrm{e}+6$ \\
128 & 86,169 & $3.30 \mathrm{e}+8$ & $6.42 \mathrm{e}+7$ & $2.17 \mathrm{e}+8$ & $5.53 \mathrm{e}+7$ & $9.97 \mathrm{e}+7$ & $4.70 \mathrm{e}+7$ \\
\hline
\end{tabular}

Table 6. Biharmonic equation, CVT meshes. Comparison of the condition number estimates obtained with the different stabilization strategies.

\begin{tabular}{r|c|cc|cc|cr}
\hline & \multirow{2}{*}{$N_{\text {dofs }}$} & \multicolumn{2}{|c|}{$\alpha_{\text {stab }}=\operatorname{Trace}\left(\mathrm{M}_{\mathrm{P}}\right) / 3$} & \multicolumn{2}{c}{$\alpha_{\text {stab }}=1 /|\mathrm{P}|$} & \multicolumn{2}{c}{$\alpha_{\text {stab }}=1 / h^{2}$} \\
$1 / h$ & $\mathrm{U}=\mathrm{I}$ & $\mathrm{U}=\mathrm{D}^{\perp}$ & $\mathrm{U}=\mathrm{I}$ & $\mathrm{U}=\mathrm{D}^{\perp}$ & $\mathrm{U}=\mathrm{I}$ & $\mathrm{U}=\mathrm{D}^{\perp}$ \\
\hline 8 & 474 & $6.42 \mathrm{e}+2$ & $1.95 \mathrm{e}+2$ & $3.22 \mathrm{e}+2$ & $1.87 \mathrm{e}+2$ & $3.40 \mathrm{e}+2$ & $1.75 \mathrm{e}+2$ \\
16 & 1704 & $8.66 \mathrm{e}+3$ & $2.82 \mathrm{e}+3$ & $4.32 \mathrm{e}+3$ & $2.09 \mathrm{e}+3$ & $3.97 \mathrm{e}+3$ & $2.11 \mathrm{e}+3$ \\
32 & 6438 & $1.55 \mathrm{e}+5$ & $4.14 \mathrm{e}+4$ & $6.77 \mathrm{e}+4$ & $2.99 \mathrm{e}+4$ & $6.52 \mathrm{e}+4$ & $3.05 \mathrm{e}+4$ \\
64 & 24,921 & $2.90 \mathrm{e}+6$ & $6.70 \mathrm{e}+5$ & $1.06 \mathrm{e}+6$ & $4.98 \mathrm{e}+5$ & $9.74 \mathrm{e}+5$ & $4.52 \mathrm{e}+5$ \\
128 & 98,724 & $4.53 \mathrm{e}+7$ & $1.14 \mathrm{e}+7$ & $1.99 \mathrm{e}+7$ & $8.43 \mathrm{e}+6$ & $1.56 \mathrm{e}+7$ & $8.00 \mathrm{e}+6$ \\
\hline
\end{tabular}


Table 7. Biharmonic equation, HEX meshes. Comparison of the condition number estimates obtained with the different stabilization strategies.

\begin{tabular}{r|c|cc|cc|cc}
\hline & & \multicolumn{2}{|c|}{$\alpha_{\text {stab }}=\operatorname{Trace}\left(\mathrm{M}_{\mathrm{P}}\right) / 3$} & \multicolumn{2}{c|}{$\alpha_{\text {stab }}=1 /|\mathrm{P}|$} & \multicolumn{2}{c}{$\alpha_{\text {stab }}=1 / h^{2}$} \\
$1 / h$ & $N^{\text {dofs }}$ & $\mathrm{U}=\mathrm{I}$ & $\mathrm{U}=\mathrm{D}^{\perp}$ & $\mathrm{U}=\mathrm{I}$ & $\mathrm{U}=\mathrm{D}^{\perp}$ & $\mathrm{U}=\mathrm{I}$ & $\mathrm{U}=\mathrm{D}^{\perp}$ \\
\hline 8 & 1371 & $1.33 \mathrm{e}+4$ & $2.68 \mathrm{e}+3$ & $6.70 \mathrm{e}+3$ & $2.20 \mathrm{e}+3$ & $4.51 \mathrm{e}+3$ & $1.99 \mathrm{e}+3$ \\
16 & 5415 & $3.52 \mathrm{e}+5$ & $5.57 \mathrm{e}+4$ & $1.94 \mathrm{e}+5$ & $4.50 \mathrm{e}+4$ & $8.87 \mathrm{e}+4$ & $3.80 \mathrm{e}+4$ \\
32 & 21303 & $5.46 \mathrm{e}+6$ & $8.81 \mathrm{e}+5$ & $2.82 \mathrm{e}+6$ & $7.07 \mathrm{e}+5$ & $1.35 \mathrm{e}+6$ & $5.89 \mathrm{e}+5$ \\
64 & 85,251 & $7.79 \mathrm{e}+7$ & $1.44 \mathrm{e}+7$ & $4.30 \mathrm{e}+7$ & $1.09 \mathrm{e}+7$ & $2.52 \mathrm{e}+7$ & $9.58 \mathrm{e}+6$ \\
128 & 343,131 & $1.04 \mathrm{e}+9$ & $2.62 \mathrm{e}+8$ & $8.72 \mathrm{e}+8$ & $2.20 \mathrm{e}+8$ & $4.26 \mathrm{e}+8$ & $1.83 \mathrm{e}+8$ \\
\hline
\end{tabular}

two cases, the performance of stabilization terms is analogous to the one observed on quadrilateral meshes, since the best accuracy is achieved by the stabilization with $\mathrm{U}=\mathrm{I}$ and $\alpha_{\text {stab }}=\operatorname{Trace}\left(\mathrm{M}_{\mathrm{P}}\right) / 3$.

\section{Conclusion}

We reviewed the construction of highly regular virtual element approximations for polyharmonic problems in two spatial dimensions, recalling the main theoretical convergence results available in the literature. Moreover, we performed a set of new two-dimensional numerical tests to investigate how different stabilizations in the formulation of the VEM affect the solver performance in terms of condition number of the resulting linear system and accuracy of the approximation schemes. For the discretization of the Poisson equation, our numerical results show that the choice of the stabilization has an almost negligible effect on condition numbers and accuracy. On the other hand, the numerical results that we obtained for the biharmonic equation shows that the choice of the stabilization may affect significantly the accuracy of approximation. This effect may be even more pronounced for $p_{1}>2$ and requires further investigation. The best overall performance in our tests is provided by the so-called dofi-dofi stabilization. On the basis of the results obtained regarding the conditioning of the highly regular VEM matrices, we also believe that it is worth of future investigations the development of effective preconditioners for VEM approximations of high-order elliptic equations.

\section{Acknowledgments}

The authors are extremely grateful to the referees for their constructive comments which have greatly contributed to the improvement of the paper. The authors are members of INdAM-GNCS. PFA and MV acknowledge the financial support of PRIN research grant no. 201744KLJL "Virtual Element Methods: Analysis and Application" funded by MIUR. GM acknowledges the financial support of the ERC Project CHANGE, which has received funding from the European Research Council under the European Union's Horizon 2020 research and innovation program (grant agreement no. 694515) 


\section{References}

1. B. Ahmad, A. Alsaedi, F. Brezzi, L. D. Marini and A. Russo, Equivalent projectors for virtual element methods, Comput. Math. Appl. 66 (2013) 376-391.

2. F. Aldakheel, B. Hudobivnik, A. Hussein and P. Wriggers, Phase-field modeling of brittle fracture using an efficient virtual element scheme, Comput. Methods Appl. Mech. Eng. 341 (2018) 443-466.

3. V. Anaya, M. Bendahmane, D. Mora and M. Sepúveda, A virtual element method for a nonlocal FitzHugh-Nagumo model of cardiac electrophysiology, IMA J. Numer. Anal. 40 (2019) 1544-1576.

4. P. F. Antonietti, L. Beirão da Veiga, S. Scacchi and M. Verani, A $C^{1}$ virtual element method for the Cahn-Hilliard equation with polygonal meshes, SIAM J. Numer. Anal. 54 (2016) 34-56.

5. P. F. Antonietti, S. Bertoluzza, D. Prada and M. Verani, The virtual element method for a minimal surface problem, Calcolo 57 Paper No. 39 (2020).

6. P. F. Antonietti, M. Bruggi, S. Scacchi and M. Verani, On the virtual element method for topology optimization on polygonal meshes: A numerical study, Comput. Math. Appl. 74 (2017) 1091-1109.

7. P. F. Antonietti, S. Giani and P. Houston, $h p$-version composite discontinuous Galerkin methods for elliptic problems on complicated domains, SIAM J. Sci. Comput. 35 (2013) A1417-A1439.

8. P. F. Antonietti, G. Manzini, I. Mazzieri, H. Mourad and M. Verani, The arbitraryorder virtual element method for linear elastodynamics models: Convergence, stability and dispersion-dissipation analysis, Internat. J. Numer. Methods Eng. 122 (2021) 934-971.

9. P. F. Antonietti, G. Manzini and M. Verani, The fully nonconforming virtual element method for biharmonic problems, Math. Models Methods Appl. Sci. 28 (2018) 387407.

10. P. F. Antonietti, G. Manzini and M. Verani, The conforming virtual element method for polyharmonic problems, Comput. Math. Appl. 79 (2020) 2021-2034.

11. P. F. Antonietti, L. Mascotto and M. Verani, A multigrid algorithm for the $p$ version of the virtual element method, ESAIM Math. Model. Numer. Anal. 52 (2018) $337-364$.

12. J. H. Argyris, I. Fried and D. W. Scharpf, The TUBA family of plate elements for the matrix displacement method, Aeronaut. J. R. Aeronaut. Soc. 72 (1968) 701-709.

13. E. Artioli, S. de Miranda, C. Lovadina and L. Patruno, A stress/displacement virtual element method for plane elasticity problems, Comput. Methods Appl. Mech. Eng. 325 (2017) 155-174.

14. E. Artioli, S. de Miranda, C. Lovadina and L. Patruno, A family of virtual element methods for plane elasticity problems based on the Hellinger-Reissner principle, Comput. Methods Appl. Mech. Eng. 340 (2018) 978-999.

15. B. Ayuso de Dios, K. Lipnikov and G. Manzini, The non-conforming virtual element method, ESAIM Math. Model. Numer. 50 (2016) 879-904.

16. I. Babuška and J. Osborn, Eigenvalue problems, in Handbook of Numerical Analysis, Vol. II (North-Holland, 1991), pp. 641-787.

17. L. Beirão da Veiga, F. Brezzi, A. Cangiani, G. Manzini, L. D. Marini and A. Russo, Basic principles of virtual element methods, Math. Models Methods Appl. Sci. 23 (2013) 199-214.

18. L. Beirão da Veiga, F. Brezzi, L. D. Marini and A. Russo, H(div) and H(curl)conforming VEM, Numer. Math. 133 (2016) 303-332. 
19. L. Beirão da Veiga, F. Brezzi, L. D. Marini and A. Russo, Mixed virtual element methods for general second-order elliptic problems on polygonal meshes, ESAIM: Math. Model. Numer. Anal. 50 (2016) 727-747.

20. L. Beirão da Veiga, F. Brezzi, L. D. Marini and A. Russo, Virtual element methods for general second-order elliptic problems on polygonal meshes, Math. Models Methods Appl. Sci. 26 (2016) 729-750.

21. L. Beirão da Veiga, F. Dassi and A. Russo, A $C^{1}$ virtual element method on polyhedral meshes, Comput. Math. Appl. 79 (2020) 1936-1955.

22. L. Beirão da Veiga, K. Lipnikov and G. Manzini, The Mimetic Finite Difference Method, Modeling, Simulations and Applications, Vol. 11, 1st edn. (Springer, 2014).

23. L. Beirão da Veiga, C. Lovadina and A. Russo, Stability analysis for the virtual element method, Math. Models Methods Appl. Sci. 27 (2017) 2557-2594.

24. L. Beirão da Veiga, C. Lovadina and G. Vacca, Divergence free virtual elements for the Stokes problem on polygonal meshes, ESAIM: Math. Model. Numer. Anal. 51 (2017) 509-535.

25. L. Beirão da Veiga, C. Lovadina and G. Vacca, Virtual elements for the Navier-Stokes problem on polygonal meshes, SIAM J. Numer. Anal. 56 (2018) 1210-1242.

26. L. Beirão da Veiga and G. Manzini, A virtual element method with arbitrary regularity, IMA J. Numer. Anal. 34 (2014) 782-799.

27. L. Beirão da Veiga and G. Manzini, Residual a posteriori error estimation for the virtual element method for elliptic problems, ESAIM Math. Model. Numer. Anal. 49 (2015) 577-599.

28. K. Bell, A refined triangular plate bending finite element, Int. J. Numer. Methods Eng. 1 (1969) 101-122.

29. M. F. Benedetto, S. Berrone and A. Borio, The virtual element method for underground flow situations in fractured data, in Advances in Discretization Methods, SEMA SIMAI Springer Series, Vol. 12 (Springer, 2016), pp. 167-186.

30. M. F. Benedetto, S. Berrone, S. Pieraccini and S. Scialò, The virtual element method for discrete fracture network simulations, Comput. Methods Appl. Mech. Eng. 280 (2014) 135-156.

31. E. Benvenuti, A. Chiozzi, G. Manzini and N. Sukumar, Extended virtual element method for the Laplace problem with singularities and discontinuities, Comput. Methods Appl. Mech. Eng. 356 (2019) 571-597.

32. H. Blum and R. Rannacher, On the boundary value problem of the biharmonic operator on domains with angular corners, Math. Methods Appl. Sci. 2 (1980) 556-581.

33. S. C. Brenner and L.-Y. Sung, Virtual enriching operators, Calcolo 56 (2019) 1-25.

34. F. Brezzi, R. S. Falk and L. D. Marini, Basic principles of mixed virtual element methods, ESAIM Math. Model. Numer. Anal. 48 (2014) 1227-1240.

35. F. Brezzi and L. D. Marini, Virtual element methods for plate bending problems, Comput. Methods Appl. Mech. Eng. 253 (2013) 455-462.

36. A. Cangiani, Z. Dong, E. H. Georgoulis and P. Houston, hp-Version Discontinuous Galerkin Methods on Polygonal and Polyhedral Meshes, Springer Briefs in Mathematics (Springer, 2017).

37. O. Certik, F. Gardini, G. Manzini and G. Vacca, The virtual element method for eigenvalue problems with potential terms on polytopic meshes, Appl. Math. 63 (2018) 333-365.

38. L. Chen and J. Huang, Some error analysis on virtual element methods, Calcolo 55 Paper No. 5 (2018) 23.

39. L. Chen and X. Huang, Nonconforming virtual element method for $2 m$ th order partial differential equations in $\mathbb{R}^{n}$, Math. Comput. 89 (2020) 1711-1744. 
40. H. Chi, A. Pereira, I. F. Menezes and G. H. Paulino, Virtual element method (VEM)based topology optimization: An integrated framework, Struct. Multidiscip. Optim. 62 (2020) 1089-1114.

41. C. Chinosi and L. D. Marini, Virtual element method for fourth-order problems: $L^{2}$ estimates, Comput. Math. Appl. 72 (2016) 1959-1967.

42. P. G. Ciarlet, The finite element method for elliptic problems, Class. Appl. Math. 40 (2002) 1-511.

43. R. W. Clough and J. L. Tocher, Finite Element Stiffness Matrices for Analysis of Plates in Bending, in Proc. Conf. on Matrix Methods in Structural Mechanics, WrightPatterson Air Force Base, Ohio, October 1965, pp. 515-545.

44. B. Cockburn, B. Dong and J. Guzmán, A superconvergent LDG-hybridizable Galerkin method for second-order elliptic problems, Math. Comput. 77 (2008) 1887-1916.

45. F. Dassi and L. Mascotto, Exploring high-order three dimensional virtual elements: Bases and stabilizations, Comput. Math. Appl. 75 (2018) 3379-3401.

46. A. Dedner and A. Hodson, Robust nonconforming virtual element methods for general fourth-order problems with varying coefficients, preprint (2020), arXiv 2008.01617.

47. D. A. Di Pietro and J. Droniou, The Hybrid High-Order Method for Polytopal Meshes: Design, Analysis, and Applications, Modeling, Simulation and Applications (Springer, 2020).

48. F. Gazzola, H.-C. Grunau and G. Sweers, Polyharmonic Boundary Value Problems: Positivity Preserving and Nonlinear Higher-Order Elliptic Equations in Bounded Domains, Lecture Notes in Mathematics, Vol. 1991 (Springer-Verlag, 2010).

49. G. H. Golub and C. F. Van Loan, Matrix Computations, Johns Hopkins Studies in the Mathematical Sciences, 3rd edn. (Johns Hopkins Univ. Press, 1996).

50. J. $\mathrm{Hu}, \mathrm{T}$. Lin and $\mathrm{Q}$. $\mathrm{Wu}, \mathrm{A}$ construction of $C^{r}$ conforming finite element spaces in any dimension, preprint (2021), arXiv:2103.14924.

51. J. Hu and S. Zhang, The minimal conforming $H^{k}$ finite element spaces on $\mathbb{R}^{n}$ rectangular grids, Math. Comput. 84 (2015) 563-579.

52. X. Huang, Nonconforming virtual element method for $2 m$ th order partial differential equations in $\mathbb{R}^{n}$ with $m>n$, Calcolo 57 Paper No. 42 (2020) 38.

53. X. Huang, $H^{m}$-conforming virtual elements in arbitrary dimension, preprint (2021), arXiv:2105.12973.

54. M. Li, J. Zhao, C. Huang and S. Chen, Conforming and nonconforming vems for the fourth-order reaction-subdiffusion equation: A unified framework, IMA J. Numer. Anal. (2021), drab030, https://doi.org/10.1093/imanum/drab030.

55. C. Lovadina, D. Mora and I. Velásquez, A virtual element method for the von Kármán equations, ESAIM Math. Model. Numer. Anal. 55 (2021) 533-560.

56. L. Mascotto, Ill-conditioning in the virtual element method: Stabilizations and bases, Numer. Methods Partial Differential Equations 34 (2018) 1258-1281.

57. L. Mascotto, I. Perugia and A. Pichler, A nonconforming Trefftz virtual element method for the Helmholtz problem, Math. Models Methods Appl. Sci. 29 (2019) 16191656.

58. L. Mascotto, I. Perugia and A. Pichler, A nonconforming Trefftz virtual element method for the Helmholtz problem: Numerical aspects, Comput. Methods Appl. Mech. Eng. 347 (2019) 445-476.

59. MATLAB. version 9.8.0 (R2020a). (The MathWorks Inc., 2020).

60. D. Mora, G. Rivera and I. Velásquez, A virtual element method for the vibration problem of Kirchhoff plates, ESAIM Math. Model. Numer. Anal. 52 (2018) 1437-1456.

61. D. Mora and A. Silgado, A $C^{1}$ virtual element method for the stationary quasigeostrophic equations of the ocean, Comput. Math. Appl. (2021), ISSN 0898-1221, https://doi.org/10.1016/j.camwa.2021.05.022. 
62. D. Mora and I. Velásquez, A virtual element method for the transmission eigenvalue problem, Math. Models Methods Appl. Sci. 28 (2018) 2803-2831.

63. D. Mora and I. Velásquez, Virtual element for the buckling problem of Kirchhoff-Love plates, Comput. Methods Appl. Mech. Eng. 360 (2020) 22.

64. K. Park, H. Chi and G. H. Paulino, On nonconvex meshes for elastodynamics using virtual element methods with explicit time integration, Comput. Methods Appl. Mech. Eng. 356 (2019) 669-684.

65. K. Park, H. Chi and G. H. Paulino, Numerical recipes for elastodynamic virtual element methods with explicit time integration, Internat. J. Numer. Methods Eng. 121 (2020) 1-31.

66. I. Perugia, P. Pietra and A. Russo, A plane wave virtual element method for the Helmholtz problem, ESAIM Math. Model. Numer. 50 (2016) 783-808.

67. N. Sukumar and A. Tabarraei, Conforming polygonal finite elements, Internat. $J$. Numer. Methods Eng. 61 (2004) 2045-2066.

68. F. Wang and H. Wei, Virtual element methods for the obstacle problem, IMA J. Numer. Anal. 40 (2018) 708-728.

69. F. Wang and J. Zhao, Conforming and nonconforming virtual element methods for a Kirchhoff plate contact problem, IMA J. Numer. Anal. 41 (2021) 1496-1521.

70. P. Wriggers, W. T. Rust and B. D. Reddy, A virtual element method for contact, Comput. Mech. 58 (2016) 1039-1050.

71. S. Zhang, A family of 3D continuously differentiable finite elements on tetrahedral grids, Appl. Numer. Math. 59 (2009) 219-233.

72. S. Zhang, A family of differentiable finite elements on simplicial grids in four space dimensions, Math. Numer. Sin. 38 (2016) 309-324.

73. J. Zhao, S. Chen and B. Zhang, The nonconforming virtual element method for plate bending problems, Math. Models Methods Appl. Sci. 26 (2016) 1671-1687.

74. J. Zhao, B. Zhang, S. Chen and S. Mao, The Morley-type virtual element for plate bending problems, J. Sci. Comput. 76 (2018) 610-629. 